\title{
Localization and chiral symmetry in three flavor domain wall QCD
}

\author{
David J. Antonio, ${ }^{1}$ Kenneth C. Bowler, ${ }^{1}$ Peter A. Boyle, ${ }^{1}$ Norman H. Christ, ${ }^{2}$ Michael A. Clark, ${ }^{3}$ Saul D. Cohen, ${ }^{2}$ \\ Chris Dawson, ${ }^{4}$ Alistair Hart, ${ }^{1}$ Balint Joó ${ }^{1,5}$ Chulwoo Jung, ${ }^{6}$ Richard D. Kenway, ${ }^{1}$ Shu Li,${ }^{2}$ Meifeng Lin, ${ }^{2}$ \\ Robert D. Mawhinney, ${ }^{2}$ Christopher M. Maynard, ${ }^{7}$ Shigemi Ohta, ${ }^{4,8,9}$ Robert J. Tweedie, ${ }^{1}$ and Azusa Yamaguchi ${ }^{10}$
}

(RBC and UKQCD Collaborations)*

\author{
${ }^{1}$ SUPA, School of Physics, The University of Edinburgh, Edinburgh EH9 3JZ, United Kingdom \\ ${ }^{2}$ Physics Department, Columbia University, New York, New York 10027, USA \\ ${ }^{3}$ Center for Computational Science, Boston University, 3 Cummington Street, Boston, Massachusetts 02215, USA \\ ${ }^{4}$ RIKEN-BNL Research Center, Brookhaven National Laboratory, Upton, New York 11973, USA \\ ${ }^{5}$ Jefferson Laboratory MS 12H2, 12000 Jefferson Avenue, Newport News, Virginia 23606, USA \\ ${ }^{6}$ Physics Department, Brookhaven National Laboratory, Upton, New York 11973, USA \\ ${ }^{7}$ EPCC, School of Physics, The University of Edinburgh, Edinburgh EH9 3JZ, United Kingdom \\ ${ }^{8}$ Institute of Particle and Nuclear Studies, KEK, Tsukuba, 305-0801, Japan \\ ${ }^{9}$ Physics Department, Sokendai Graduate University of Advanced Studies, Hayama, Kanagawa 240-0193, Japan \\ ${ }^{10}$ SUPA, Department of Physics \& Astronomy, University of Glasgow, Glasgow G12 8QQ, United Kingdom
}

(Received 18 May 2007; published 22 January 2008)

\begin{abstract}
We present results for the dependence of the residual mass of domain wall fermions on the size of the fifth dimension and its relation to the density and localization properties of low-lying eigenvectors of the corresponding Hermitian Wilson Dirac operator in three flavor domain wall QCD. Using the DBW2 and Iwasaki gauge actions, we generate ensembles of configurations with a $16^{3} \times 32$ space-time volume and an extent of 8 in the fifth dimension for the sea quarks. We demonstrate the existence of a regime where the degree of locality, the size of chiral symmetry breaking, and the rate of topology change can be acceptable for inverse lattice spacings $a^{-1} \geq 1.6 \mathrm{GeV}$, enabling a programme of simulations of $2+1$ flavor QCD to be conducted safely in this region of parameter space.
\end{abstract}

DOI: 10.1103/PhysRevD.77.014509

\section{INTRODUCTION}

In this paper we present a study of three closely related quantities that are important for the successful application of the domain wall fermion (DWF) formulation to full QCD calculations with up, down, and strange flavors of light quarks. The first of these is the size of the explicit chiral symmetry breaking that occurs for domain wall fermions due to the finite extent of the lattice in the fifth dimension. This is usually characterized by the residual mass, $m_{\text {res }}$, whose dependence on the gauge action, gauge coupling, and the extent of the fifth dimension is discussed.

The second topic covers the spectral properties of the four-dimensional Hermitian Wilson Dirac operator evaluated at large, negative mass, $-M_{5}$. All current formulations of lattice chiral symmetry make use of a negativemass (Hermitian) Wilson Dirac operator, $H_{W}=\gamma_{5} D_{W}$, and intellectually derive from the Kaplan approach [1], whether by taking an analytic limit as in the overlap operator [2], or by directly simulating a finite fifth dimension [3] of size $L_{s}$. Auxiliary information on the nature of the spectrum of $H_{W}$ is required to have confidence that a simulation at fixed lattice spacing lies in the universality

*CU-TP-1160, Edinburgh 2007/3, RBRC-649, KEK-TH-1138, BNL-NT 06/29.
PACS numbers: 11.15.Ha, 11.30.Rd, 12.38.Aw, 12.38.Gc

class of QCD. This topic is closely connected to the properties of the Aoki phase of the related operator $D_{W}$ [4]. If a chiral fermion calculation is attempted for a gauge action and a negative fermion mass that is too close to the Aoki phase, undesirable nonlocality or enhanced explicit chiral symmetry breaking may result. Thus, we will examine both the density of the low-lying eigenmodes of $H_{W}$ and their locality properties.

Finally, we examine the ergodicity of the sampling of topological charge that results for these choices of gauge action and negative Wilson Dirac mass using the rational hybrid Monte Carlo method of Clark and Kennedy [5,6]. Since a change in the topological charge of an evolving lattice configuration is expected to be accompanied by a zero mode of $H_{W}$, a choice of the gauge action which suppresses such zero modes will inhibit topology change, possibly rendering the Monte Carlo sampling nonergodic in practice.

Based on these studies, we conclude that lattice QCD calculations with $2+1$ flavors of light quarks are possible with adequate locality, small explicit chiral symmetry breaking, and adequate sampling of topological charge for inverse lattice spacings of $1.6 \mathrm{GeV}$ or larger. In particular, the rapid decrease in the rate of change of topological charge for the DBW2 action with decreasing lattice spacing favors the choice of Iwasaki action - a choice that has 
been adopted for the large-scale ensemble generation of the RBC and UKQCD collaborations using the QCDOC computers [7-9] at the RIKEN-BNL Research Center and the University of Edinburgh.

The structure of this paper is as follows. In Sec. II we describe the Aoki phase of the Wilson Dirac operator in light of recent research and the connection to the spectrum and localization of eigenmodes of $H_{W}$. In Sec. III we review the importance of the spectrum of $H_{W}$ for lattice formulations that accurately realize chiral symmetry. The explicit breaking of chiral symmetry that results from finite $L_{s}$ for domain wall fermions is discussed in Sec. IV and the expected dependence of the residual mass on $L_{s}$ motivated. In Sec. V we describe the details of the gauge and DWF actions used in our simulations, while in Sec. VI we present a microscopic study of the low-lying eigenvalues and eigenmodes of $H_{W}$ on our ensembles. In Sec. VII we present a study of the dependence on $L_{s}$ of the residual mass for valence quarks on each of the (fixed sea quark $L_{s}$ ) ensembles. The $L_{s}$ dependence of these results are fitted to the form motivated in Sec. IV, and conclusions about the low-lying spectrum of the transfer matrix in the fifth dimension are drawn and related to our results from Sec. VI. Finally, in Sec. VIII, we examine the dependence of the topological charge with evolving Monte Carlo time and demonstrate the correlation between reduced DWF chiral symmetry breaking and a suppression in the rate of change of topological charge. The Appendix summarizes the transfer matrix formalism of Furman and Shamir, which is used extensively in the residual mass discussion of Sec. IV.

\section{AOKI PHASE DIAGRAM OF LATTICE QCD}

The properties of lattice chiral fermions are intimately connected with the properties of the Hermitian Wilson Dirac operator $H_{W}$ for negative mass $-M_{5}$. Of specific interest is the "supercritical" region, $-8 \leq-M_{5} \leq 0$, in which both $H_{W}$ and $D_{W}$ can have zero eigenvalues. This region shows a surprisingly rich and interesting phase structure, first conjectured and described by Aoki [4,1012] in both the quenched and dynamical cases [13].

The Aoki phase was originally defined as a region in which a nonvanishing pionic condensate spontaneously breaks flavor and parity for the two-flavor dynamical case, with an associated flavor nonsinglet Goldstone pion. In the quenched case, if one examines Green's functions made from a single flavor of quark, (discrete) parity is spontaneously broken. The propagator for a flavor-singlet pion describes a massless state at the critical line separating the normal from the Aoki phase, but which becomes massive within the interior of the Aoki phase. For Green's functions containing two quark flavors, connected by a vanishing flavor-breaking mass term, parity and flavor are broken by the pionic condensate $\bar{\psi} \gamma_{5} \tau^{a} \psi$, and massless, flavored Goldstone modes exist inside the Aoki phase.
Since the Hermitian Wilson Dirac operator obeys a Banks-Casher relation, a nonzero density of near-zero modes is associated with this condensate in both the quenched and dynamical cases. This nonzero density is a strong-coupling effect, arising from the disorder in the gauge fields which characterizes the strong-coupling limit. As the coupling becomes weak, the gauge field becomes more ordered and such a density of near-zero modes is expected to disappear [14], except for values of $M_{5}$ where the free Dirac operator itself has zero modes, $-M_{5}=0$, $-2,-4,-6$, and -8 .

However, the picture suggested by the simple summary above is not complete. It was observed $[12,15]$ that the interior of the quenched Aoki phase consists of two qualitatively different regions bounded by a critical line $g_{c}\left(-M_{5}\right)$. Above $g_{c}\left(-M_{5}\right)$ near-zero modes exist which are delocalized, while below $g_{c}\left(-M_{5}\right)$ only localized nearzero modes appear. In both regions the pionic condensate and density of near-zero modes are nonzero, but their localization properties differ fundamentally.

This picture was substantially refined and solidified by Golterman et al. [16-19] who, in close analogy with consequences of randomness in condensed matter physics, introduced to QCD the concept of a nonzero mobility edge $\lambda_{c}$, as the critical eigenvalue of $H_{W}$ above which eigenstates are extended and below which all eigenstates are localized. They also applied the McKane and Stone localization escape from Goldstone's theorem to lattice QCD at nonzero lattice spacing in the quenched case. Specifically, they showed that two quenched flavors of Wilson fermion with a nonzero mobility edge can display a spontaneous breaking of a continuous symmetry without a corresponding Goldstone boson. This observation is key to the correct functioning of all the various formulations of Kaplan fermions wherever the kernel $H_{W}$ has a nonzero density of low modes.

This qualitative picture is displayed in Fig. 1. Following conventional terminology, we refer to the Aoki phase as that region of this diagram in which $\left\langle\bar{\psi} \gamma^{5} \tau^{a} \psi\right\rangle \neq 0$ and $g^{2}>g_{c}^{2}\left(-M_{5}\right)$. In this region the coupling is sufficiently large that the mobility edge is zero and long-range correlations result from massless flavor-nonsinglet pions. The near-zero modes of $H_{W}$ are delocalized. This is a dangerous region for chiral fermions, with lattice artifacts producing unphysical, long-distance correlations. For weaker coupling $g^{2} \leq g_{c}^{2}\left(-M_{5}\right)$, these long-distance correlations have disappeared. The Wilson Dirac eigenvectors with low eigenvalues are localized on the lattice scale and all delocalized modes have eigenvalues which, if expressed in physical units, are at least as large as $\lambda_{c}\left(-M_{5}\right) / a$. These delocalized low modes do not introduce unphysical nonlocality into the corresponding formulation of lattice chiral fermions but, as discussed in the next section, they enhance the explicit violation of chiral symmetry for domain wall fermions with finite $L_{s}$. 


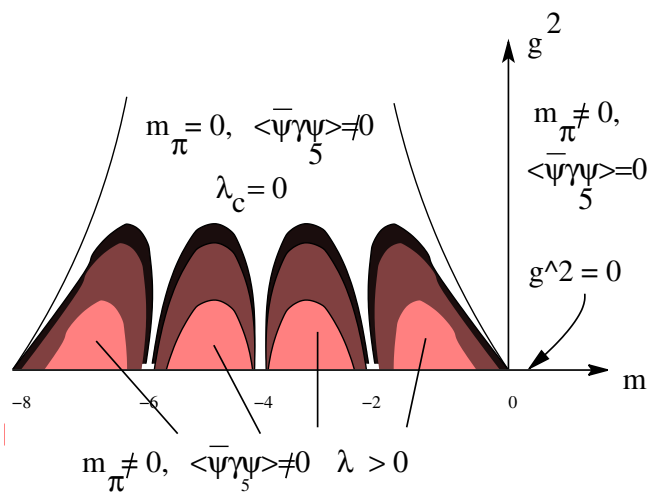

FIG. 1 (color online). Schematic diagram of the quenched Aoki phase. A pionic condensate and corresponding nonzero density of near-zero modes is developed throughout most of the negative mass region. In the colored sectors, a nonzero mobility edge is developed and the contours could equally well represent either decreasing low mode density, decreasing pionic condensate, or increasing $\lambda_{c}$ as one moves towards the continuum limit at $g^{2}=0$.

\section{LOW-LYING SPECTRUM OF $H_{W}$ AND LATTICE CHIRAL FERMIONS}

We now discuss in more detail the relation between the spectrum of the Hermitian Wilson Dirac operator $H_{W}$ and the properties of domain wall fermions. Let us first address the issue of locality. While the domain wall Dirac operator contains only nearest-neighbor couplings in five dimensions (i.e. it is ultralocal), the four-dimensional effective low-energy theory which it produces may contain unphysical nonlocality. One approach to investigating this question is to examine the locality of the overlap operator that results in the $L_{s} \rightarrow \infty$ limit [20,21]. This was done by Hernandez et al. [22] using $H_{W}$ as the kernel in the sign function of the overlap operator. They showed that a gap in the spectrum of the eigenstates of $H_{W}$ guarantees locality of the resulting overlap operator. The proof relies on the ultralocal nature of $H_{W}$ implying ultralocality of a finite polynomial of $H_{W}$. The "Shamir" kernel $K_{S}$ for the overlap operator that corresponds to the $L_{s}=\infty$ limit of domain wall fermions can be taken as $K_{S}=H_{W}\left(2+D_{W}\right)^{-1}$ [23-25] and it is not manifestly ultralocal. However, the operator $2+D_{W}$ in the denominator of $K_{S}$ is the Wilson Dirac operator with a $2-M_{5}$ mass term, and hence (provided $M_{5}<2$ ) is outside the supercritical region ensuring that this kernel is exponentially localized. The Hernandez et al. proof is therefore applicable to domain wall fermions under the modification that a finite polynomial of an exponentially local operator is also exponentially local.

However, as discussed above, the assumed gap in the spectrum of $H_{W}$ is neither expected nor observed in lattice QCD, at least for the range of couplings in which calculations are normally performed. Fortunately, as argued by Golterman et al. [16], the detailed properties of the Wilson Dirac operator in the supercritical region described above are sufficient to imply that the overlap operator is appropriately local [provided we are outside of the Aoki phase and $\left.\lambda_{c}\left(-M_{5}, g\right)>0\right]$. If, as is the case in this picture, one assumes that the spectrum of extended states shows a gap (the region $0 \leq \lambda \leq \lambda_{c}$ ) so that any eigenstates within the gap are localized, then the resulting overlap operator will also be localized (even though the density of low modes is nonzero). This is supported by numerical evidence at currently affordable couplings.

Finally, we examine a second difficulty faced by domain wall fermions and related approaches, which is closely connected with these low-lying modes of $H_{W}$. The residual chiral symmetry breaking effects seen at finite $L_{s}$ for domain wall fermions depend in detail on the densities and sizes of the modes of the Hermitian matrix $H_{T}$. This is reviewed in the Appendix, and $H_{T}$ is defined in Eq. (A15). $H_{T}$ is used to construct the Fock-space transfer matrix in the fifth dimension, $T$, defined in Eq. (A13). This transfer matrix describes explicitly how the left and right walls are coupled when $L_{s}$ is finite. While the relation between the operators $H_{W}$ and $H_{T}$ is somewhat complex, it can be shown that their zero modes coincide [3], and also that $-\log T \equiv H_{T}=2 \tanh ^{-1} K_{S}$, where $K_{S}=\frac{H_{W}}{2+D_{W}}$ [23-25]. Further, the corresponding approximation to the overlap operator, and its $L_{s} \rightarrow \infty$ limit are [20]

$\begin{aligned} D_{\mathrm{ov}}^{\mathrm{approx}} & =\frac{1}{2}\left[1+m+(1-m) \gamma_{5} \tanh \left(\frac{L_{s}}{2} H_{T}\right)\right] \\ \rightarrow D_{\mathrm{ov}} & \left.=\frac{1}{2}\left[1+m+(1-m) \gamma_{5} \operatorname{sgn} H_{T}\right)\right],\end{aligned}$

or equivalently,

$$
\begin{aligned}
& D_{\mathrm{ov}}^{\text {approx }}=\frac{1}{2}\left[1+m+(1-m) \gamma_{5} \tanh \left(L_{s} \tanh ^{-1} K_{S}\right)\right] \\
& \left.\rightarrow D_{\mathrm{ov}}=\frac{1}{2}\left[1+m+(1-m) \gamma_{5} \operatorname{sgn} K_{S}\right)\right] .
\end{aligned}
$$

The equality of the operators $\operatorname{sgn} H_{T}$ and $\operatorname{sgn} K_{S}$ follows easily from the relation $H_{T}=2 \tanh ^{-1} K_{S}$ and the recognition that the inverse hyperbolic tangent is a function which preserves the sign of its argument.

Thus, the near-zero modes of $H_{W}$ discussed above are expected to correspond to near-zero modes of $H_{T}$, modes which (in the absence of an explicit mass term) will dominate the coupling of the left- and right-handed sectors of low-energy domain wall QCD. Similarly, the mobility edge structure of $H_{W}$ is also expected to describe $H_{T}$. As is exploited in the next section, this implies a simple structure for the asymptotic $L_{s}$ dependence of the residual mass. The near-zero localized modes will give a power behavior $\left(1 / L_{s}\right)$, while the extended modes above the mobility edge give an exponential falloff $\left(e^{-\lambda_{c} L_{s}} / L_{s}\right)$. This dependence will be used as one of several diagnostics to investigate the nature of the $H_{T}$ spectrum in our simulations.

The strong-coupling behavior of the Wilson Dirac operator does not leave one at liberty to simulate QCD at 
arbitrarily coarse lattice spacings. For a given lattice action, the phase boundary where the gap in the spectrum of extended states vanishes defines the coarsest lattice spacing at which the formulation makes sense and implies a minimum cost that must be paid to have the formulation under control. The phase boundary is action dependent and, in practice, we will wish to stay well within the phase such that the mobility edge is not small.

We have performed the first numerical study of the localization of the Hermitian Wilson Dirac operator with three mass degenerate flavors of light dynamical quarks and have demonstrated that a programme of $2+1$ flavor dynamical DWF simulations of QCD is rendered affordable by our current QCDOC computer systems. These considerations are directly relevant for all chirally symmetric lattice QCD formulations, and we shall later illustrate the smooth connection between finite $L_{s}$ and the $L_{s} \rightarrow \infty$ overlap limit with numerical data for the plaquette. Our work has been presented at Lattice 2005 [26], and later, while this paper was being completed, a broadly similar study was presented at Lattice 2006 using dynamical overlap fermions [27].

\section{RESIDUAL MASS}

In this section we discuss the residual mass in some detail, including a careful discussion of its dependence on $L_{s}$ as predicted by the transfer matrix analysis of Furman and Shamir. As we will demonstrate and has been worked out previously [16-19,26,28], this dependence on $L_{s}$ permits the effects of both the localized near-zero modes and the extended modes above the mobility edge to be recognized. This gives useful information about the general character of chiral symmetry breaking as well as an understanding of the origin of the residual mass itself.

In the limit of small lattice spacing, or equivalently weak gauge coupling, the spectrum of the domain wall Dirac operator for a typical gauge background is expected to have a physical, four-dimensional component with eigenvalues $\lambda \sim \Lambda_{\mathrm{QCD}}$ as well as unphysical, five-dimensional states with $\lambda \sim 1 / a$. The low-lying modes permit the accurate simulation of $\mathrm{QCD}$, while the large eigenvalues are lattice artifacts similar to, but more numerous than, the unphysical large eigenvalues found in other lattice fermion formulations. Ideally, the physical four-dimensional states will be bound near the $s=0$ and $s=L_{s}-1$ walls. To the extent that $L_{s}$ is large, there should be little mixing between the left-handed chiral states localized on the left $(s=0)$ wall and the right-handed chiral states localized on the right $\left(s=L_{s}-1\right)$ wall.

The low-energy properties of this approximately chiral theory can be described by an effective Lagrangian, $\mathcal{L}_{\text {eff }}$. If effects coming from nonzero lattice spacing or arising from the overlap between the left- and right-handed states are neglected, this effective Lagrangian will be precisely that of QCD. The corrections coming from these effects can be described by adding extra operators to $\mathcal{L}_{\text {eff }}$. The only relevant operator of this sort is a dimension-3 mass term. The next most important term is the familiar dimension-5 Sheikholeslami-Wohlert term. Thus,

$$
\mathcal{L}_{\text {eff }}=\mathcal{L}_{\mathrm{QCD}}+m_{\mathrm{res}} \bar{q} q+c_{5} \bar{q} \sigma^{\mu \nu} F^{\mu \nu} q+\ldots
$$

In this paper we focus on the coefficient, $m_{\text {res }}$, of this residual mass term as an important measure of the violation of chiral symmetry arising from the finite extent $\left(L_{s}\right)$ in the fifth dimension. In this section, we will discuss the dependence of $m_{\text {res }}$ on $L_{s}$ by using the transfer matrix formalism of Furman and Shamir [3], described in the Appendix, which is a prerequisite for those unfamiliar with this formalism.

\section{A. Transfer matrix in the fifth dimension}

Using the transfer matrix approach, the approximate chiral symmetry of domain wall fermions is realized by writing a general Green's function by semi-independent products of left- and right-handed operators. As is described in the Appendix, if we arrange the field variables appearing in a general Green's function by segregating the left- and right-handed fields into two products, $\mathcal{O}_{L}\left[P_{L} q, \bar{q} P_{R}\right]$ and $\mathcal{O}_{R}\left[P_{R} q, \bar{q} P_{L}\right]$, then we can write

$$
\begin{aligned}
& \left\langle\mathcal{O}_{L}\left[P_{L} q, \bar{q} P_{R}\right] \mathcal{O}_{R}\left[P_{R} q, \bar{q} P_{L}\right]\right\rangle_{L_{s}} \\
& \quad=Z\left(L_{s}\right) \operatorname{tr}\left\{\mathcal{O}_{L}\left[P_{L} \hat{a},-\hat{a}^{\dagger} P_{R}\right] \hat{T}^{L_{s}} \mathcal{O}_{R}\left[P_{R} \hat{a}, \hat{a}^{\dagger} P_{L}\right] \mathcal{O}\left(m_{f}\right)\right\} .
\end{aligned}
$$

The left-hand side of this equation is a general, DWF Green's function defined as a Grassmann integral over the five-dimensional domain wall field variables $\bar{\Psi}(x, s)$ and $\Psi(x, s)$ using the usual DWF action, $S_{F}=$ $-\bar{\Psi} D^{\mathrm{DWF}} \Psi$. Here the Grassmann fields $\bar{\Psi}(x, s)$ and $\Psi(x, s)$ are functions of a space-time coordinate $x$ and fifth-dimensional coordinate $s, 0 \leq s \leq L_{s}-1$. The domain wall Dirac operator $D^{\mathrm{DWF}}$ is that of Shamir [29], and Furman and Shamir [3], and in our notation is given by

$$
\begin{aligned}
D_{x, s ; x^{\prime}, s^{\prime}}^{\mathrm{DWF}}( & \left.M_{5}, m_{f}\right)=\delta_{s, s^{\prime}} D_{x, x^{\prime}}^{\|}\left(M_{5}\right)+\delta_{x, x^{\prime}} D_{s, s^{\prime}}^{\perp}\left(m_{f}\right), \\
D_{x, x^{\prime}}^{\|}\left(M_{5}\right)= & \frac{1}{2} \sum_{\mu=1}^{4}\left[\left(1-\gamma_{\mu}\right) U_{x, \mu} \delta_{x+\hat{\mu}, x^{\prime}}\right. \\
& \left.+\left(1+\gamma_{\mu}\right) U_{x^{\prime}, \mu}^{\dagger} \delta_{x-\hat{\mu}, x^{\prime}}\right]+\left(M_{5}-4\right) \delta_{x, x^{\prime}}, \\
D_{s, s^{\prime}}^{\perp}\left(m_{f}\right)= & \frac{1}{2}\left[\left(1-\gamma_{5}\right) \delta_{s+1, s^{\prime}}+\left(1+\gamma_{5}\right) \delta_{s-1, s^{\prime}}-2 \delta_{s, s^{\prime}}\right] \\
& -\frac{m_{f}}{2}\left[\left(1-\gamma_{5}\right) \delta_{s, L_{s}-1} \delta_{0, s^{\prime}}\right. \\
& \left.+\left(1+\gamma_{5}\right) \delta_{s, 0} \delta_{L_{s}-1, s^{\prime}}\right] .
\end{aligned}
$$

The physical four-dimensional Grassmann fields $q(x)$ and 
$\bar{q}(x)$ are constructed from the five-dimensional fields $\bar{\Psi}(x, s)$ and $\Psi(x, s)$ according to Eqs. (A16) and (A17).

The right-hand side (RHS) of Eq. (4) is the trace of a product of operators acting on a many-particle Fock space. In contrast to the usual field theory setting, these particles are located in a four-dimensional coordinate space. Thus, the fields $a_{x}$ and $a_{x}^{\dagger}$ depend on the space-time position $x$, as well as other spin and color indices which are not shown. These operators, as well as the transfer matrix $\hat{T}$, and mass operator $\mathcal{O}\left(m_{f}\right)$, are defined in the Appendix. The factor $Z\left(L_{s}\right)$ is an $L_{s}$-dependent normalization factor. The mass $M_{5}$ is the domain wall height. The mass $m_{f}$ is the bare mass of the physical fermions.

In the limit in which $m_{f}=0$ and $L_{s} \rightarrow \infty, \hat{T}^{L_{s}}$ is viewed as projecting onto the state with the largest eigenvalue (normalized to be unity), and the right-hand side of Eq. (4) becomes the product of two completely independent matrix elements corresponding to isolated left- and right-handed theories [see Eq. (A35)]. This exact chiral symmetry is violated by the operator $\mathcal{O}\left(m_{f}\right)$ which represents the explicit mass term and mixes the left- and righthanded factors. Similarly, the corrections to the asymptotic limit $\hat{T}^{L_{s}} \rightarrow\left|0_{H}\right\rangle\left\langle 0_{H}\right|$ of Eq. (A36) introduce chiral symmetry breaking arising from finite $L_{s}$.

In the above discussion, and that to follow, we are ignoring the effects of anomalous chiral symmetry breaking. Such effects require modifications to our discussion for background gauge fields with nonzero Pontryagin index. For such gauge fields the state $\left|0_{H}\right\rangle$ will carry a fermion number different from that of $\left|0_{S}\right\rangle$, thereby introducing flavor-singlet chirality correlations between the otherwise independent left- and right-hand factors in the first term on the right-hand side of Eq. (4) [30]. In the interest of simplicity, we do not consider such gauge configurations here. However, the effects of these configurations do not alter our conclusions.

\section{B. Residual mass and the transfer matrix}

We will now explicitly study the first corrections to the large $L_{s}$ limit of $\hat{T}^{L_{s}}$ in Eq. (4) where, for clarity, we set $m_{f}=0$ and use the relation $\mathcal{O}\left(m_{f}=0\right)=\left|0_{S}\right\rangle\left\langle 0_{S}\right|$ discussed in the Appendix:

$$
\begin{aligned}
& \left\langle\mathcal{O}_{L}\left[P_{L} q, \bar{q} P_{R}\right] \mathcal{O}_{R}\left[P_{R} q, \bar{q} P_{L}\right]\right\rangle_{L_{s}} \\
& =Z\left(L_{s}\right)\left\langle 0_{S}\right| \mathcal{O}_{L}\left[P_{L} \hat{a},-\hat{a}^{\dagger} P_{R}\right]\left\{\left|0_{H}\right\rangle\left\langle 0_{H}\right|\right. \\
& \quad+\sum_{k^{+}=1}^{N^{+}} e^{-E_{k^{+}}^{+} L_{s}} \hat{o}_{k^{+}}^{\dagger}\left|0_{H}\right\rangle\left\langle 0_{H}\left|\hat{o}_{k^{+}}+\sum_{k^{-}=1}^{N^{-}} e^{-E_{k^{-}} L_{s}} \hat{p}_{k^{-}}^{\dagger}\right| 0_{H}\right\rangle \\
& \left.\quad \times\left\langle 0_{H}\right| \hat{p}_{k^{-}}\right\} \mathcal{O}_{R}\left[P_{R} \hat{a}, \hat{a}^{\dagger} P_{L}\right]\left|0_{S}\right\rangle
\end{aligned}
$$

Here the leading term, the projection operator $\left|0_{H}\right\rangle\left\langle 0_{H}\right|$, divides the Green's function into two independent factors demonstrating the separate flavored chiral symmetry of the left- and right-handed fermions. The first correction permits quark number \pm 1 exchanges between these two otherwise independent sectors. This suggests that the second and third terms in Eq. (8) should be interpreted at low energies as the residual mass operator $m_{\text {res }} \bar{q} q$ expressed in this five-dimensional language.

A relation between this residual mass term and the second and third terms in the operator in curly brackets in Eq. (8) can be obtained if we express the operators $\hat{o}_{k}$ and $\hat{p}_{k}$ in terms of the conventional four-dimensional field $\hat{a}_{x}$, inverting Eq. (A25):

$$
\begin{aligned}
& -\sum_{n} m_{\mathrm{res}} \bar{q}\left(x_{n}\right) q\left(x_{n}\right) \rightarrow m_{\mathrm{res}} \sum_{n}\left\{\left(\hat{a}_{x_{n}}^{\dagger} P_{R}\right)_{\alpha}\left|0_{H}\right\rangle\left\langle 0_{H}\right|\left(P_{R} \hat{a}_{x_{n}}\right)_{\alpha}\right. \\
& \left.+\left(P_{L} \hat{a}_{x_{n}}\right)_{\alpha}\left|0_{H}\right\rangle\left\langle 0_{H}\right|\left(\hat{a}_{x_{n}}^{\dagger} P_{L}\right)_{\alpha}\right\} \\
& \stackrel{?}{\approx} \sum_{k} \sum_{n, n^{\prime}} \phi_{k \alpha}^{+}\left(x_{n}\right) \phi_{k \beta}^{+}\left(y_{n^{\prime}}\right)^{*} e^{-L_{s} E_{k}^{+}}\left(\hat{a}_{x_{n}}^{\dagger} P_{R}\right)_{\alpha}\left|0_{H}\right\rangle \\
& \times\left\langle 0_{H}\right|\left(P_{R} \hat{a}_{y_{n^{\prime}}}\right)_{\beta} \\
& +\sum_{k} \sum_{n, n^{\prime}} \phi_{k \alpha}^{-}\left(x_{n}\right)^{*} \phi_{k \beta}^{-}\left(y_{n^{\prime}}\right) e^{-L_{s} E_{k}^{-}}\left(P_{L} \hat{a}_{x_{n}}\right)_{\alpha}\left|0_{H}\right\rangle \\
& \times\left\langle 0_{H}\right|\left(\hat{a}_{y_{n^{\prime}}}^{\dagger} P_{L}\right)_{\beta} \text {. }
\end{aligned}
$$

Here the RHS of Eq. (9) is the usual residual mass term, $-m_{\text {res }} \bar{q} q$, written in the transfer matrix language [see Eq. (A19)], while the expression in Eq. (10) is a version of the leading order contribution from the second and third terms in Eq. (8). The projection operators $P_{R}$ and $P_{L}$ can be introduced into the right-hand side of Eq. (10) because the other chirality will vanish in the limit $m_{f}=0$ [31].

We can justify this relation and estimate $m_{\text {res }}$ if we assume that the sums $\sum_{k} \phi_{k}^{ \pm}\left(x_{n}\right)^{\dagger} \phi_{k}^{ \pm}\left(y_{n^{\prime}}\right)$ are localized on the long-distance scale at which $m_{\text {res }}$ is defined:

$$
\begin{aligned}
K_{\alpha, \beta}(x, y) & =\sum_{k} \phi_{k, \alpha}^{ \pm}(x)^{\dagger} \phi_{k, \beta}^{ \pm}(y) e^{-L_{s} E_{k}^{ \pm}} \\
& \approx m_{\mathrm{res}} \delta^{4}(x-y) \delta_{\alpha, \beta},
\end{aligned}
$$

where $\alpha$ and $\beta$ are both spin and color indices, and we assume that the diagonal spin/color structure will appear and any dependence on the label \pm will disappear when a volume and/or gauge average is performed. We can then approximate $m_{\text {res }}=\frac{1}{12} \int d^{4} x K_{\alpha, \alpha}(x, y) \approx \frac{1}{12} R^{4} K_{\alpha, \alpha}(y, y)$, where the "radius" $R$ estimates the small region in $x$ that contributes and summation is performed over the repeated spin and color index $\alpha$. Finally, $K(y, y)$ and hence $m_{\text {res }}$ can be determined by integrating over $y$ and using the orthonormality of the eigenfunctions $\phi_{k \alpha}^{ \pm}(y)$ :

$$
m_{\text {res }}=\frac{R^{4}}{12} K_{\alpha, \alpha}(y, y)=\frac{R^{4}}{12 L^{3} L_{t}} \int d^{4} y K_{\alpha, \alpha}(y, y)
$$




$$
\begin{gathered}
=\frac{R^{4}}{12 L^{3} L_{t}} \sum_{k} e^{-L_{s} E_{k}^{ \pm}}=R^{4} \int_{0}^{\infty} d \lambda \rho(\lambda) e^{-L_{s} \lambda} \\
\sim R_{e}^{4} \rho_{e}\left(\lambda_{c}\right) \frac{e^{-\lambda_{c} L_{s}}}{L_{s}}+R_{l}^{4} \rho_{l}(0) \frac{1}{L_{s}} .
\end{gathered}
$$

Here $\rho(\lambda)$ on the RHS of Eq. (13) is the density of eigenvalues of $H_{T}$ per unit space-time volume, color, and spin. The final Eq. (14) is a generalization of Eq. (13) displaying the expected contributions of extended $(e)$ and localized $(l)$ modes with possibly different radius parameters, $R_{l}$ and $R_{e}$. Equation (14) should be viewed as a theoretically motivated model for the large $L_{s}$ behavior of the residual mass where $R_{l}$ is the radius of the localized states with eigenvalue close to zero. The radius associated with the extended states, $R_{e}$, arises from the limited spatial resolution provided by a sum over extended states resulting from the high-momentum cutoff intrinsic to the lattice formulation. This suggests $R_{e} \approx a$. In this final equation we have also taken the limit of large $L_{s}$ to simplify the integral over $\lambda$. This is the "standard" result for the dependence of $m_{\text {res }}$ on $L_{s}$, although the factor of $1 / L_{s}$ is usually missing from the left-most term. We will use Eq. (14) elsewhere in this paper to interpret the dependence of $m_{\text {res }}$ on $L_{s}$.

\section{Residual mass and the five-dimensional axial current}

While the above discussion provides a direct connection between corrections to the $L_{s} \rightarrow \infty$ limit and the residual mass, it does not give a simple way to compute $m_{\text {res }}$. This is typically done using a partially conserved five-dimensional axial current introduced by Furman and Shamir [3]:

$$
\mathcal{A}_{\mu}^{b}(x)=\sum_{s=0}^{L_{s}-1} \operatorname{sign}\left(s-\frac{L_{s}-1}{2}\right) j_{\mu}^{b}(x, s),
$$

where the current $j_{\mu}^{b}(x, s)$ is defined by

$$
\begin{aligned}
j_{\mu}^{b}(x, s)= & \frac{1}{2}\left[\bar{\Psi}(x+\hat{\mu}, s)\left(1+\gamma_{\mu}\right) U_{x+\mu, \mu}^{\dagger} t^{b} \Psi(x, s)\right. \\
& \left.-\bar{\Psi}(x, s)\left(1-\gamma_{\mu}\right) U_{x, \mu} t^{b} \Psi(x+\mu, s)\right]
\end{aligned}
$$

and $b$ is a flavor index and $t^{b}$ is a generator of the flavor symmetry. The equations of motion imply the conservation law

$$
\Delta_{\mu} \mathcal{A}_{\mu}^{b}(x)=2 m_{f} \bar{q}(x) t^{b} \gamma^{5} q(x)+J_{5 q}^{b}(x),
$$

where $J_{5 q}^{b}$ is defined in Eq. (A20) and we define $\left(\Delta_{\mu} f\right) \times$ $(x)=f(x)-f(x-\hat{\mu})$.

In the limit of large $L_{s}$ and small lattice spacing, the extra $J_{5 q}^{b}$ term in the conservation law in Eq. (17) should describe the chiral asymmetry of the leading dimension-3 term in the domain wall fermion effective Lagrangian, $m_{\text {res }} \bar{q} q$ :

$$
J_{5 q}^{b} \approx 2 m_{\mathrm{res}} \bar{q} t^{b} \gamma^{5} q
$$

This equation can be used directly to determine $m_{\text {res }}$ by evaluating the ratio of the matrix elements of $J_{5 q}^{b}$ and $\bar{q} t^{b} \gamma^{5} q$. In this paper, we use the ratio of pion correlation functions,

$$
m_{\mathrm{res}}=\frac{\left\langle J_{5 q}^{b}(x) \bar{q} t^{b} \gamma^{5} q(y)\right\rangle}{\left\langle 2 \bar{q} t^{b} \gamma^{5} q(x) \bar{q} t^{b} \gamma^{5} q(y)\right\rangle},
$$

evaluated for $|x-y| \gg a$ to determine $m_{\text {res }}$ numerically.

As a consistency check, we should demonstrate that the definitions of $m_{\text {res }}$ given in Eqs. (10) and (18) agree. We begin by generalizing Eq. (A22) to express a general Green's function of $J_{5 q}^{b}(z)$ with other physical fields, in a form in which the latter are separated into the left- and right-handed factors $\mathcal{O}_{L}\left[P_{L} q, \bar{q} P_{R}\right]$ and $\mathcal{O}_{R}\left[P_{R} q, \bar{q} P_{L}\right]$ :

$$
\begin{aligned}
& \left\langle\mathcal{O}_{L}\left[P_{L} q, \bar{q} P_{R}\right] J_{5 q}^{b}(z) \mathcal{O}_{R}\left[P_{R} q, \bar{q} P_{L}\right]\right\rangle \\
& =Z\left(L_{s}\right)\left\langle 0_{S}\right| \mathcal{O}_{L}\left[P_{L} \hat{a},-\hat{a}^{\dagger} P_{R}\right] \hat{T}^{L_{s} / 2} \hat{a}_{z}^{\dagger} t^{b} \hat{a}_{z} \\
& \quad \times \hat{T}^{L_{s} / 2} \mathcal{O}_{R}\left[P_{R} \hat{a}, \hat{a}^{\dagger} P_{R}\right]\left|0_{S}\right\rangle .
\end{aligned}
$$

Since we wish to compare with Eqs. (9) and (10), we can simplify this expression by integrating the position variable $z$ over space-time:

$$
\begin{gathered}
Z\left(L_{s}\right)\left\langle 0_{S}\right| \mathcal{O}_{L}\left[P_{L} \hat{a},-\hat{a}^{\dagger} P_{R}\right] \hat{T}^{L_{s} / 2}\left\{\sum_{k^{+}=1}^{N^{+}} \hat{o}_{k^{+}}^{\dagger} t^{b} \hat{o}_{k^{+}}\right. \\
\left.-\sum_{k^{-}=1}^{N^{-}} \hat{p}_{k^{-}}^{\dagger} t^{b} \hat{p}_{k^{-}}\right\} \hat{T}^{L_{s} / 2} \mathcal{O}_{R}\left[P_{R} \hat{a}, \hat{a}^{\dagger} P_{R}\right]\left|0_{S}\right\rangle .
\end{gathered}
$$

Here we have also replaced the operators $\hat{a}_{x}$ and $\hat{a}_{x}^{\dagger}$ with those creating eigenstates of $\hat{T}$, using the definition in Eq. (A25) and the orthogonality relations obeyed by the coefficients $\phi_{k^{ \pm}}^{ \pm}$. Next, we can evaluate the factors $\hat{T}^{L_{s} / 2}$ in the limit of large $L_{s}$ using Eq. (A36). The leading contribution to Eq. (21) results when the next leading contribution to $\hat{T}^{L_{s} / 2}$ [the second and third terms in Eq. (A36)] is substituted for each factor:

$$
\begin{aligned}
& Z\left(L_{s}\right)\left\langle 0_{S}\right| \mathcal{O}_{L}\left[P_{L} \hat{a},-\hat{a}^{\dagger} P_{R}\right]\left(\sum_{k_{1}^{+}=1}^{N^{+}} e^{-E_{k_{1}^{+}}^{+}\left(L_{s} / 2\right)} \hat{o}_{k_{1}^{+}}^{\dagger}\left|0_{H}\right\rangle\right. \\
& \left.\quad \times\left\langle 0_{H}\left|\hat{o}_{k_{1}^{+}}+\sum_{k_{1}^{-}=1}^{N^{-}} e^{-E_{k_{1}^{-}}^{-}\left(L_{s} / 2\right)} \hat{p}_{k_{1}^{-}}^{\dagger}\right| 0_{H}\right\rangle\left\langle 0_{H}\right| \hat{p}_{k_{1}^{-}}\right) \\
& \quad \times\left\{\sum_{k^{+}=1}^{N^{+}} \hat{o}_{k^{+}}^{\dagger} t^{b} \hat{o}_{k^{+}}-\sum_{k^{-}=1}^{N^{-}} \hat{p}_{k^{-}}^{\dagger} t^{b} \hat{p}_{k^{-}}\right\} \\
& \quad \times\left(\sum_{k_{2}^{+}=1}^{N^{+}} e^{-E_{k_{2}^{+}}^{+}\left(L_{s} / 2\right)} \hat{o}_{k_{2}^{+}}^{\dagger}\left|0_{H}\right\rangle\left\langle 0_{H}\right| \hat{o}_{k_{2}^{+}}\right. \\
& \left.\quad+\sum_{k_{2}^{-}=1}^{N^{-}} e^{-E_{k_{2}^{-}}^{-\left(L_{s} / 2\right)}} \hat{p}_{k_{2}^{-}}^{\dagger}\left|0_{H}\right\rangle\left\langle 0_{H}\right| \hat{p}_{k_{2}^{-}}\right) \mathcal{O}_{R}\left[P_{R} \hat{a}, \hat{a}^{\dagger} P_{L}\right]\left|0_{S}\right\rangle
\end{aligned}
$$


Exploiting the simple form of the operator in the curly brackets, Eq. (22) can be considerably simplified, yielding

$$
\begin{gathered}
Z\left(L_{s}\right)\left\langle 0_{S}\right| \mathcal{O}_{L}\left[P_{L} \hat{a},-\hat{a}^{\dagger} P_{R}\right]\left\{\sum_{k^{+}=1}^{N^{+}} e^{-E_{k^{+}}^{+} L_{s}} \hat{o}_{k^{+}}^{\dagger}\left|0_{H}\right\rangle t^{b}\left\langle 0_{H}\right| \hat{o}_{k^{+}}\right. \\
\left.-\sum_{k^{-}=1}^{N^{-}} e^{-E_{k^{-}}^{-} L_{s}} \hat{p}_{k^{-}}^{\dagger}\left|0_{H}\right\rangle t^{b}\left\langle 0_{H}\right| \hat{p}_{k^{-}}\right\} \mathcal{O}_{R}\left[P_{R} \hat{a}, \hat{a}^{\dagger} P_{L}\right]\left|0_{S}\right\rangle
\end{gathered}
$$

where the flavor generator $t^{b}$ acts on the suppressed flavor indices carried by the operators $\hat{o}_{k^{+}}^{\dagger}, \hat{o}_{k^{+}}, \hat{p}_{k^{-}}^{\dagger}$, and $\hat{p}_{k^{-}}$. Now we can directly compare the operator appearing in curly brackets in Eq. (23) (which should reduce to the operator $2 m_{\text {res }} \bar{q} t^{b} \gamma^{5} q$ at low energies) with the second and third terms of the operator in curly brackets in Eq. (8) (which we have shown does reduce to the operator $m_{\text {res }} \bar{q} q$ in this limit). These two operators are directly related at first order in $\delta$ by an infinitesimal chiral transformation, where operators in the left-hand factor transform as

$$
\hat{o}_{k^{+}}^{\dagger} \rightarrow \hat{o}_{k^{+}}^{\dagger}\left(1+i \delta t^{b}\right), \quad \hat{p}_{k^{-}}^{\dagger} \rightarrow \hat{p}_{k^{-}}^{\dagger}\left(1-i \delta t^{b}\right),
$$

while operators in the right-hand factor transform as

$$
\hat{o}_{k^{+}} \rightarrow\left(1+i \delta t^{b}\right) \hat{o}_{k^{+}}, \quad \hat{p}_{k^{-}} \rightarrow\left(1-i \delta t^{b}\right) \hat{p}_{k^{-}} .
$$

Since this relation between the operators in curly brackets in Eqs. (8) and (23) will continue to hold in the longdistance limit, each limiting quantity, $2 m_{\text {res }} \bar{q} t^{b} \gamma^{5} q$ and $m_{\text {res }} \bar{q} q$, must contain the same value of $m_{\text {res }}$. Thus, the residual mass defined in Eq. (19) agrees with that appearing in the residual mass operator deduced from the large $L_{s}$ limit of the transfer matrix for the case $m_{f}=0$. This demonstrates the expected consistency with a Symanzik effective Lagrangian description of the low-energy effects of mixing between the $s=0$ and $s=L_{s}-1$ walls.

\section{LATTICE ACTIONS AND ENSEMBLES}

Our simulations have been performed with three flavors of dynamical DWF for a class of improved gauge actions. Our notation is the same as in [32-34], which we briefly review here. The partition function we simulate is

$$
Z=\int[d U] \int \prod_{i=1}^{3}\left[d \Psi_{i} d \bar{\Psi}_{i}\right] \int \prod_{i=1}^{3}\left[d \Phi_{\mathrm{PV}, i}^{\dagger} d \Phi_{\mathrm{PV}, i}\right] e^{-S},
$$

where the index $i$ runs over the up, down, and strange quarks. The Pauli-Villars fields $\Phi_{\mathrm{PV}, i}$ are needed to cancel the bulk infinity that would be produced by DWF as $L_{s} \rightarrow$ $\infty$. In particular, the total action is

$$
S=S_{G}(U)+S_{F}(\bar{\Psi}, \Psi, U)+S_{\mathrm{PV}}\left(\Phi^{\dagger}, \Phi, U\right) .
$$

The gauge actions we consider are of the form

$$
\begin{aligned}
S_{G}[U]= & -\frac{\beta}{3}\left[\left(1-8 c_{1}\right) \sum_{x ; \mu<\nu} P[U]_{x, \mu \nu}\right. \\
& \left.+c_{1} \sum_{x ; \mu \neq \nu} R[U]_{x, \mu \nu}\right]
\end{aligned}
$$

where $P[U]_{x, \mu \nu}$ and $R[U]_{x, \mu \nu}$ represent the real part of the trace of the path ordered product of link variables around the $1 \times 1$ plaquette and the $1 \times 2$ rectangle, respectively, in the $\mu, \nu$ plane at the point $x$, and $\beta \equiv 6 / g^{2}$ with $g$ the bare quark-gluon coupling. We use two common choices for $c_{1}$ :

(i) the Iwasaki action which sets $c_{1}=-0.331[35,36]$, and

(ii) the DBW2 action which has $c_{1}=-1.4069[37,38]$. Both are known to reduce the residual mass very effectively in the quenched case.

The fermion action in Eq. (27) can be expressed in a form very close to the way it appears in the 3-flavor numerical work if it is written as

$$
S_{F}=-\sum_{i=1}^{3} \bar{\Psi}_{i}\left[D_{\mathrm{DWF}}^{\dagger}\left(M_{5}, m_{i}\right) D_{\mathrm{DWF}}\left(M_{5}, m_{i}\right)\right]^{1 / 2} \Psi_{i},
$$

where $m_{i}$ is the input bare quark mass for the $i$ th light quark flavor and the DWF Dirac operator $D_{\mathrm{DWF}}$ is defined in Eq. (5). We only consider the case where all light quarks have the same value for the five-dimensional domain wall height, $M_{5}$. The action for the Pauli-Villars fields is similar, except that the quark mass $m_{i}$ is replaced by 1 , to yield

$$
S_{\mathrm{PV}}=\sum_{i=1}^{3} \Phi_{i}^{\dagger}\left[D_{\mathrm{DWF}}^{\dagger}\left(M_{5}, 1\right) D_{\mathrm{DWF}}\left(M_{5}, 1\right)\right]^{1 / 2} \Phi_{i} .
$$

The resulting determinants are suitable for simulation using a weight

$$
\prod_{i} \frac{\operatorname{det}^{1 / 2}\left[D_{\mathrm{DWF}}^{\dagger}\left(M_{5}, m_{i}\right) D_{\mathrm{DWF}}\left(M_{5}, m_{i}\right)\right]}{\operatorname{det}^{1 / 2}\left[D_{\mathrm{DWF}}^{\dagger}\left(M_{5}, 1\right) D_{\mathrm{DWF}}\left(M_{5}, 1\right)\right]} .
$$

The action may be simulated using either the inexact $R$ algorithm [39], with associated step size errors, or the exact the exact rational hybrid Monte Carlo algorithm (RHMC) of Clark and Kennedy [40,41]. For the RHMC simulations, each of the square root factors in Eq. (31) is approximated by a rational function accurate over some eigenvalue range. Thus our simulation uses a $1+1+1$ framework that happens to be mass degenerate. Where our mapping of the Aoki phase finds a clearly nonzero mobility edge, it should be relevant to our more general programme of $2+1$ flavor simulations where a chiral limit is taken in mass degenerate up/down quark masses at fixed strange mass. When written as a partial fraction expansion, each term is individually amenable to the usual HMC pseudofermionic integral (though, of course, a single pseudofermion and multimass inverter is used for all terms in the partial fraction expansion). We used the $R$ algorithm for the earlier runs in our parameter search and, when available, preferred 
TABLE I. Ensembles used in this study. We also document the trajectories used for $m_{\text {res }}$ measurements, using every tenth trajectory in the given range. The trajectory length was $\tau=0.5$ units of MD time. All ensembles used three degenerate DWF flavors of mass $m=0.04$, and $M_{5}=1.8$.

\begin{tabular}{llcr}
\hline \hline Gauge action & $\beta$ & Algorithm & Trajectories \\
\hline DBW2 & 0.72 & $\mathrm{R}$ & $800-1500$ \\
DBW2 & 0.764 & $\mathrm{R}$ & $800-1500$ \\
DBW2 & 0.78 & $\mathrm{R}$ & $1000-1600$ \\
DBW2 & 0.80 & $\mathrm{R}$ & $900-1800$ \\
DBW2 & 0.88 & $\mathrm{R}$ & $1000-3000$ \\
Iwasaki & 2.13 & RHMC & $1500-2400$ \\
Iwasaki & 2.2 & RHMC & $800-1500$ \\
Iwasaki & 2.3 & RHMC & $800-1500$ \\
\hline \hline
\end{tabular}

to use the RHMC algorithm, as documented in Table I. Our implementation of the RHMC used separate fields as stochastic estimators of the numerator and denominator of Eq. (31). We simulated all three flavors in the numerator as separate single-flavor species, and treated the Pauli-Villars fields as separately estimated two- and single-flavor pseudofermions. Thus, we did not make any explicit use of the mass degeneracy between up and down quarks (as would have been the case, for example, in two-flavor HMC).

The ensembles used in this study are a subset of those presented in [42-44], and have been used for precision valence measurements with $L_{s}=8$. We used a reduced statistic subset to perform a consistent study as we vary $L_{s}$ in the (still expensive) valence analysis. The larger statistical sample used for the $L_{s}=8$ data points in [42-44] lead to lower errors on results for $m_{\text {res }}$ than we present in this work. We include only lower precision values in this paper to keep the $L_{s}=8$ analysis identical in all systematic respects to the $L_{s}>8$ points that are unique to this work. The ensembles used in this work are listed in Table I. For the full simulation details, we refer the reader to [4244] where the entire data set is presented.

All ensemble and correlation function computations were performed using the Columbia physics system (CPS) on QCDOC computers in Edinburgh and Brookhaven. All measurements of four-dimensional eigenmodes were performed using the CHROMA physics system [45], while the five-dimensional modes were measured using CPS. The relation between these modes provides a nontrivial cross-check between code bases. Both code bases made use of the optimized Bagel QCD library [46]. Visualization was carried out using the OPENDX software package.

\section{SPECTRUM OF THE HERMITIAN WILSON DIRAC OPERATOR}

In this section we study the eigenspectrum and eigenmodes of $H_{W}$ on our ensembles. We determined up to 256 eigenvectors of $H_{W}$ on 25 configurations per ensemble, at the negative mass $M_{5}=1.8$ that was used in our domain wall action for the sea quarks. Additional eigenvalues were generated on a number of configurations as a function of $M_{5}$. Our study therefore includes the dependence of the low-lying spectrum on $M_{5}$, often called spectral flows, in Sec. VI A. We also study the spectral density of near-zero modes in Sec. VIB, the microscopic shape distribution of the low-lying modes in Sec. VIC, and the relation between modes of the Hermitian five-dimensional operator $H_{\mathrm{DWF}}=\gamma_{5} R_{5} D_{\mathrm{DWF}}$ and $H_{W}$ in Sec. VID, where $R_{5}$ is the reflection operator in the fifth dimension.

\section{A. Spectral flows}

Prior to recent work on localization, it was believed that a gap in the spectrum was required for the correct behavior of the various lattice formulations of chiral symmetry. In this context, it was believed that, by obtaining spectral flows of the lowest few modes on a "typical" configuration, the health of the formulation was indicated by a nicely opened "eye" for some range of $M_{5}$ containing the Wilson mass used in the simulation. Recent improved understanding suggests that a nonzero, but low, density of localized states exists throughout the region in which we can afford to simulate. A gap, as such, does not exist after an ensemble average and, if spectral flows from sufficiently many configurations were overlaid, the entire eye would be filled in.

However, on a small sample of configurations an open eye is indicative of a low density of low-lying modes and, we argue, a nonzero mobility edge since our results show that the spectral density grows rapidly across this edge. The flows presented here also allow comparison between our ensembles and those of previous works. These plots for the lowest modes, Figs. 2 and 3, are therefore useful information at the larger values of $\beta$ where the open eye is indicative of a nonzero lower bound for the mobility edge, as presented previously [47].

Our spectral flow results are certainly indicative of a nonzero mobility edge for $\beta \geq 0.78$ for the DBW2 gauge action and $\beta \geq 2.2$ for the Iwasaki gauge action. For smaller values of $\beta$ the spectral flows are inconclusive as no opening is displayed suggesting a nonzero density of low modes. We shall return to look at the localization structure in a more sophisticated fashion and classify these modes in Sec. VIC.

\section{B. Spectral density}

For each data set comprising $n_{\text {conf }}$ configurations, we label the $i$ th eigenvalue on the $j$ th configuration $\lambda_{i j}$. We use a somewhat simplistic binning approach to estimate the spectral density for a partitioning of the spectrum. For the $k$ th bin we estimate the density as

$$
\rho^{k}\left(\left[\lambda_{\min }^{k}+\lambda_{\max }^{k}\right] / 2\right)=\frac{1}{n_{\text {conf }}} \frac{N_{k}}{\lambda_{\max }^{k}-\lambda_{\min }^{k}},
$$



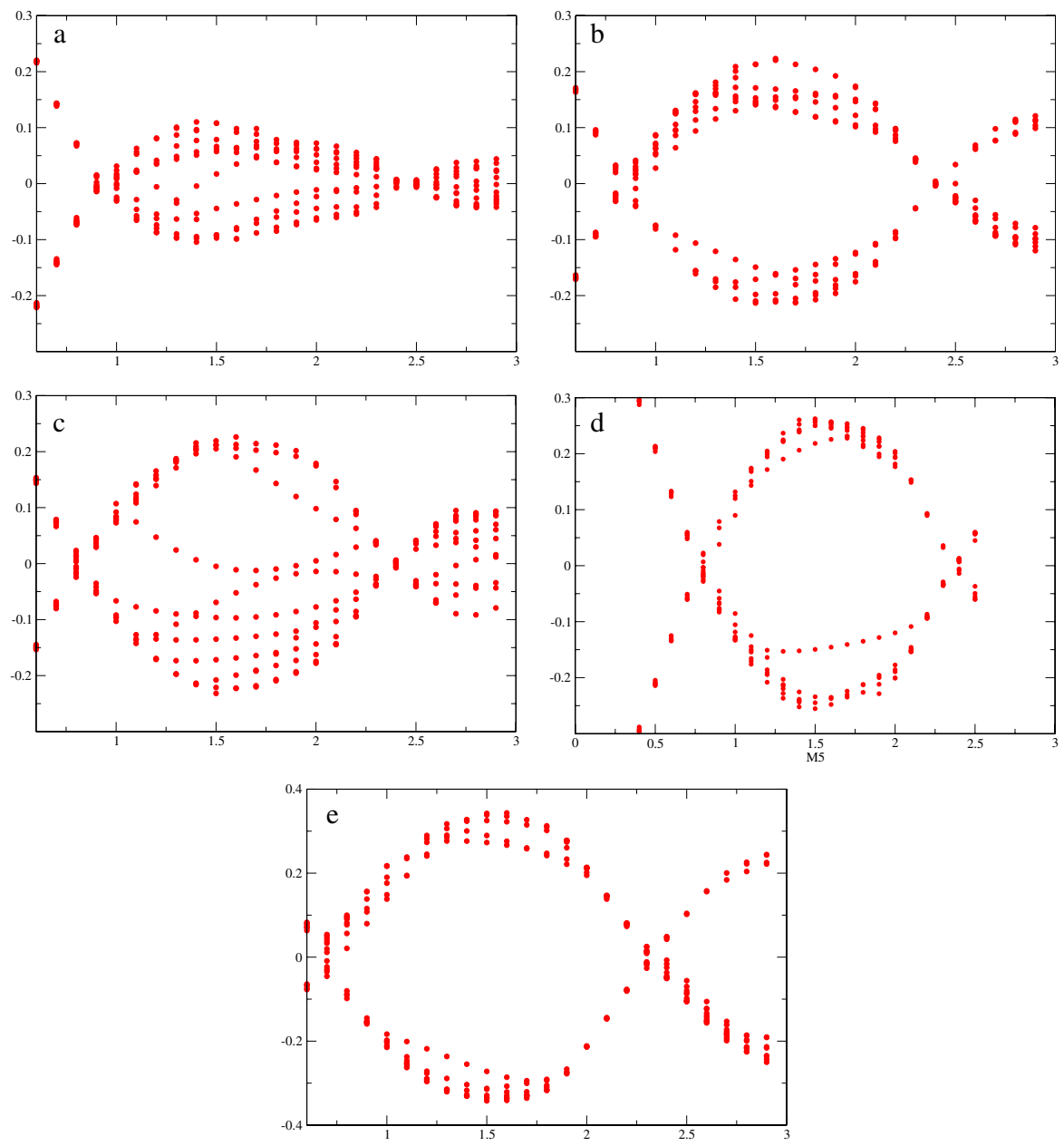

FIG. 2 (color online). Spectral flow of DBW2. The horizontal axis is the valence domain wall height, $0 \leq M_{5} \leq 3.0$, and the vertical axis is the eigenvalue of $H_{W},-0.3 \leq \lambda_{H_{W}} \leq 0.3$. From the left, $\beta=0.72, \beta=0.764$ (first row), $\beta=0.78, \beta=0.80$ (second row), and $\beta=0.88$ (third row).

where $N_{k}$ is the number of eigenvalues $\lambda_{i j} \in\left[\lambda_{\min }^{k}+\right.$ $\left.\lambda_{\max }^{k}\right], j=1, \ldots, n_{\text {conf }}$. Figure 4 displays our results for the spectral densities for our various data sets, using a fixed bin width of 0.02. Some clear, and unsurprising, trends are evident: the spectral density $\rho(0)$ is nonzero and falls rapidly as $\beta$ is increased. The quantity $\rho(0)$ is slightly higher for the Iwasaki action at $\beta=2.2$ than for DBW2 at $\beta=0.78$ which have comparable lattice spacings. The density $\rho(\lambda)$ rises rapidly, and perhaps exponentially, in $\lambda$ as we move away from zero.

\section{Microscopic study of the mobility edge}

We wish to study the size distribution of our eigenvectors, $\psi(x)$, as a function of the eigenvalue in order to test the mobility edge conjecture. This approach is novel and was first presented in [26] for dynamical DWF, with a similar approach taken in [27] for dynamical overlap. A standard measure is the inverse participation ratio,

$$
P^{-1}=\sum_{x}\left|\psi^{\dagger}(x) \psi(x)\right|^{2} .
$$

A normalized extended state will produce $P \simeq V$, while a completely localized state will produce $P \simeq 1$. Thus the (inverse) participation ratio essentially counts the number of occupied sites and, in the infinite volume limit, is a useful order parameter for delocalization transitions. However, this metric is not sensitive to the shape of a state and so makes no distinction between occupying two adjacent sites and occupying two well-separated sites, such as the illustrative nonlocal example in Fig. 5.

We want to understand how to reliably distinguish exponentially compact states from those with any degree of long-range support. In the infinite volume limit the use of the inverse participation ratio only identifies long-range correlations arising from structures whose fourdimensional volume diverges, and we seek a more robust measure.

While a better measure could be a moment of the eigenvector density, such as

$$
\sum_{x} \psi^{\dagger}(x) \psi(x)|x|
$$




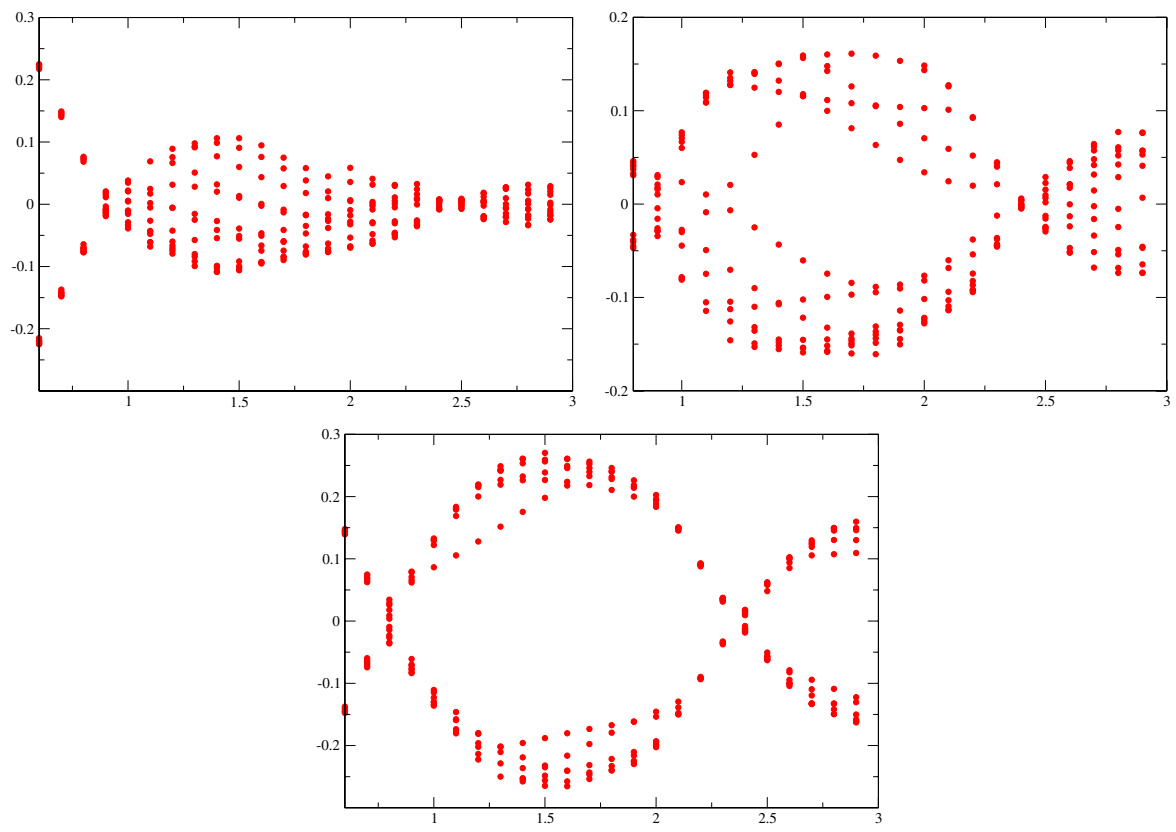

FIG. 3 (color online). Spectral flow of Iwasaki $\beta=2.13, \beta=2.2$ (first row, left to right), and $\beta=2.3$ (second row). Axes are the same as Fig. 2.

we have found empirically, by visualizing data, that near the delocalization threshold the dominant long-range correlation arises from multipeaked eigenvectors, which fall exponentially around multiple centers. We define a more robust measure of exponential localization length, which generalizes the one-dimensional case of Fig. 5 to higher dimensions as follows:

(i) The mode density is defined as $\phi(x)=\psi^{\dagger}(x) \psi(x)$.

(ii) We identify a center $x_{0}$ such that $\phi\left(x_{0}\right) \geq$ $\phi(x) \forall x \neq x_{0}$.

(iii) For every site, we define a radius $r(x)=\left|x-x_{0}\right|$

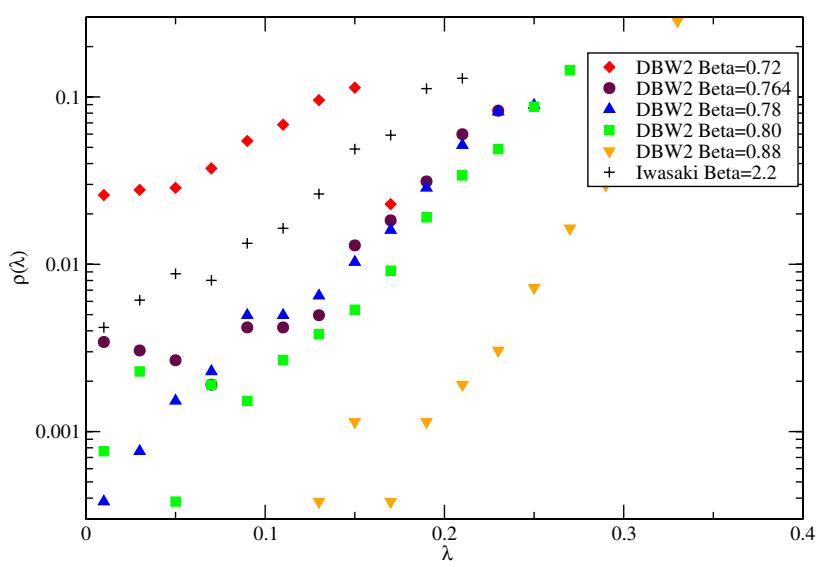

FIG. 4 (color online). Spectral density obtained on a subset of our data with no error analysis, and fixed bin width 0.02 . Thus, where the density is low the error could be large and the data should be considered only as a qualitative indication of the nature of our ensembles. using the periodic mirror image nearest to $x_{0}$.

(iv) We define an effective localization exponent $L_{\text {eff }}(x)=\frac{2 r(x)}{\log \phi\left(x_{0}\right)-\log \phi(x)}$.

(v) Finally, we define a robust localization length $L_{\text {max }}=\max _{r(x) \geq 5} L_{\text {eff }}(x)$.

As the eigenvalue approaches the mobility edge from below, we observed two clear processes by which delocalization takes place. First, the mean rate of falloff from the

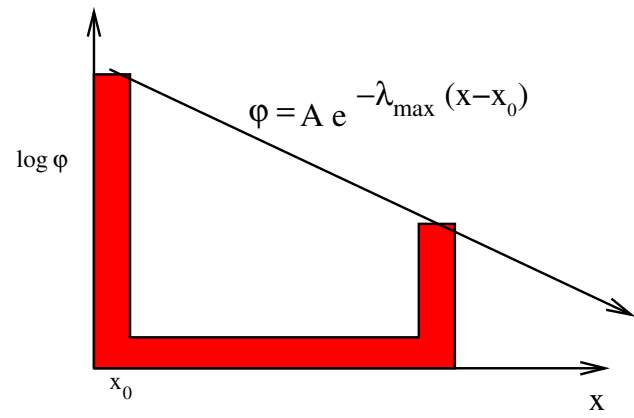

FIG. 5 (color online). Various measures can be used to define the degree of localization. The inverse participation ratio has been a popular order parameter for delocalization transitions and, while fine for that purpose, counts occupied sites without paying attention to mode shape. This one-dimensional cartoon highlights the advantages of the more robust measure employed in this paper. Given the model of exponential localization, our tactic is to find a localization exponent that forms a tight bound for the eigenvector. This measure will show the influence of satellite peaks in a robust fashion by eliminating all forms of dilution by volume average. Other useful measures that take into account the shape would include appropriately weighted moments of the density function. 


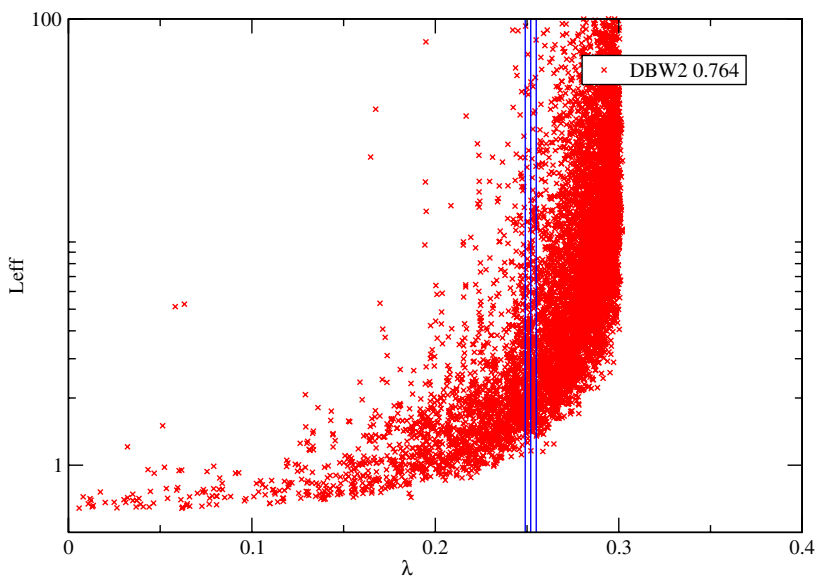

FIG. 6 (color online). Microscopic view of the mobility edge based on a scatter plot of maximal localization lengths of individual low eigenmodes of $H_{W}$. We overlay the $H_{T}$ mobility edge $\lambda_{c}$ from fits to the model for $m_{\text {res }}$, Eq. (35), and find good agreement with our diverging localization length for the DBW2 gauge action at $\beta=0.764$.

center decreases, creating much bigger states. Second, states become increasingly multicentered, with exponential falloff between a number of satellite peaks. The above definition of localization length is designed to reflect both the process of single-peak broadening and the development of multiple peaks. The inverse participation ratio would be a less robust measure of locality in that it would not differentiate two well-separated $\delta$ functions from those on two neighboring sites, or indeed would not identify a lowerdimensional extended sheet in the infinite volume. We display scatter plots of this definition of localization length for the 256 lowest eigenvectors of $H_{W}$ on 25 configurations

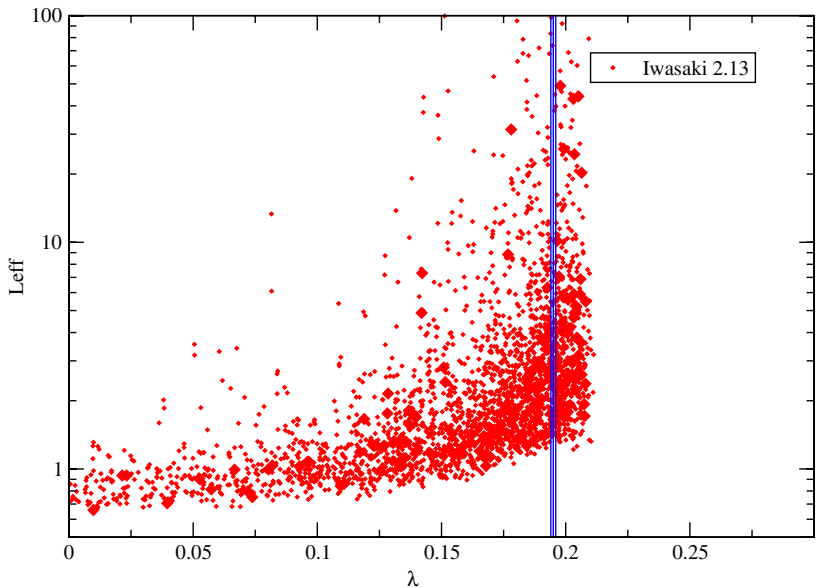

FIG. 7 (color online). Microscopic view of the mobility edge based on a scatter plot of maximal localization lengths of individual low eigenmodes of $H_{W}$. We overlay the $H_{T}$ mobility edge $\lambda_{c}$ from fits to the model for $m_{\text {res }}$, Eq. (35), and find good agreement with our diverging localization length for the Iwasaki gauge action at $\beta=2.13$. per ensemble in Figs. 6 and 7 for the DBW2 and Iwasaki gauge actions at $\beta=2.13$ and $\beta=0.764$, respectively.

\section{Relation between eigenmodes of the five- and four-dimensional operators}

We computed the lowest 10 modes of the fivedimensional Hermitian domain wall operator $H_{\mathrm{DWF}}=$ $\gamma_{5} R_{5} D_{\mathrm{DWF}}$, using the Columbia physics system, on a single configuration from a $16^{3} \times 32 \times 8$ DBW2 three flavor ensemble at $\beta=0.764$. On the same configuration, we used the lowest 256 eigenmodes $\psi_{j}(x)$ of $H_{W}$, computed using the CHROMA software package, to express the five-dimensional modes $\Psi_{i}(x, s)$ as a sum over fourdimensional modes using

$$
\Psi_{i}(x, s)=N_{s} \sum_{j} \alpha_{i j}(s) \psi_{j}(x)
$$

where

$$
N_{s}^{2}=\sum_{x} \Psi_{i}^{\dagger}(x, s) \Psi_{i}(x, s)
$$

normalizes each $s$ slice of the five-dimensional eigenvector to unity. This means that if the basis of four-dimensional eigenvectors were complete

$$
\sum_{j} \alpha_{i j}(s)^{2}=1
$$

However, since we are only using the lowest 256 eigenmodes, we expect that Eq. (32) will, at best, be true only deep in the bulk for large $L_{s}$ where low modes of the transfer matrix dominate, and only up to differences between $H_{T}$ and $H_{W}$.

We therefore computed five-dimensional modes for a valence $L_{s}=16$ (N.B. not the unitary $L_{s}=8$ case) finding that, while the basis is very much incomplete on the wall, the description it gives in the five-dimensional bulk is as much as $80 \%$ complete by the midpoint, despite the mismatch between $H_{T}$ and $H_{W}$. (This also serves as a useful cross-check between the eigensolvers in the two independent code bases.)

The coefficients $\left|\alpha_{i j}\right|^{2}$ for a typical five-dimensional chiral mode are displayed in Fig. 10, and it can be seen that only 3 low-lying modes of $H_{W}$ dominate the $s$ dependence of eigenmodes of $H_{\mathrm{DWF}}$. Thus, we clearly demonstrate from numerical data that a few localized low-lying four-dimensional modes of $H_{W}$ almost completely describe the coupling between walls for each fivedimensional mode of the Hermitian DWF operator for large $L_{s}$. As discussed, these modes occur with a low density - perhaps a "dilute gas" is an appropriate picture-but, for sufficiently large $L_{s}$, they dominate the contributions to chiral symmetry breaking due to exponential suppression of the extended modes above the mobility edge; see Eq. (14). 


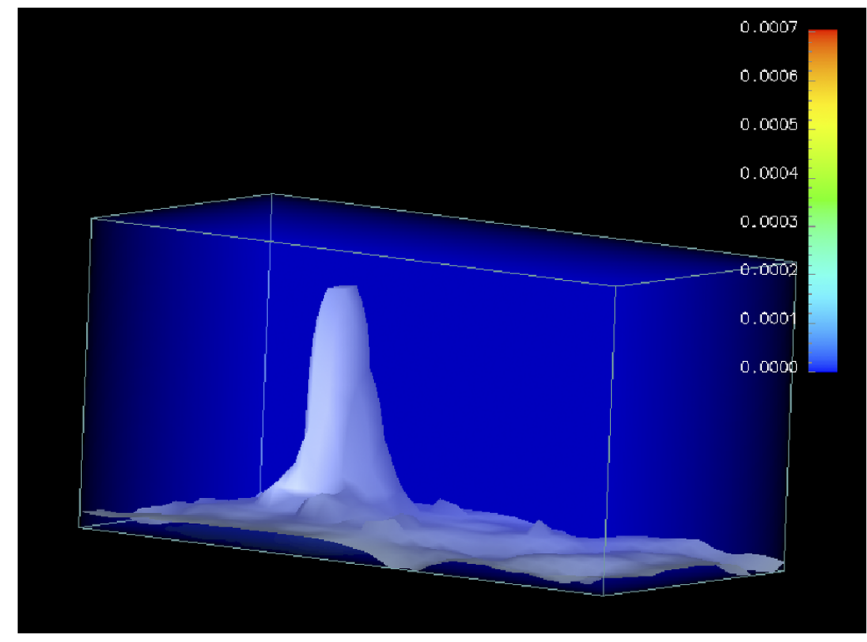

FIG. 8 (color online). We display an isosurface through the eigenvector density of a typical near-zero chiral mode of the five-dimensional operator $H_{\mathrm{DWF}}$, bound to a single wall. Here $s$ is the vertical direction, $t$ the left-right axis, and $z$ runs into the page. We show $(x, y)=(3,3)$ the eigenmode exhibits a localized incursion of the eigenmode into the fifth dimension associated with a low mode of $H_{W}$. This mechanism is responsible for one form of the contributions to chiral symmetry breaking in DWF.

The localized low modes of $H_{W}$ thus play a role in providing conduits into the bulk for chiral symmetry breaking, causing corresponding localized spikes in the correlation function for $m_{\text {res }}$. This is corroborated by the isosurfaces (produced with the OPENDX package) of con-

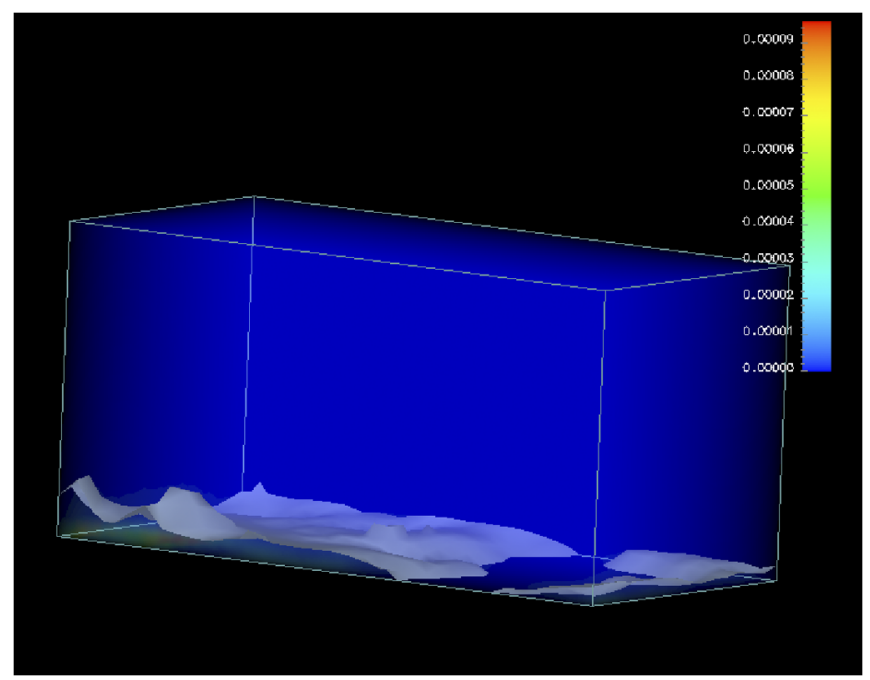

FIG. 9 (color online). We display an isosurface through the eigenvector density of the same chiral mode displayed in Fig. 8. Here $s$ is the vertical direction, $t$ the left-right axis, and $z$ runs into the page. We show $(x, y)=(11,13)$ the eigenmode exhibits a uniformly well stuck region of this mode. Compared with the previous figure, this degree of binding is the more common case for this typical eigenvector, and one has to search spatially to find any localized incursion.

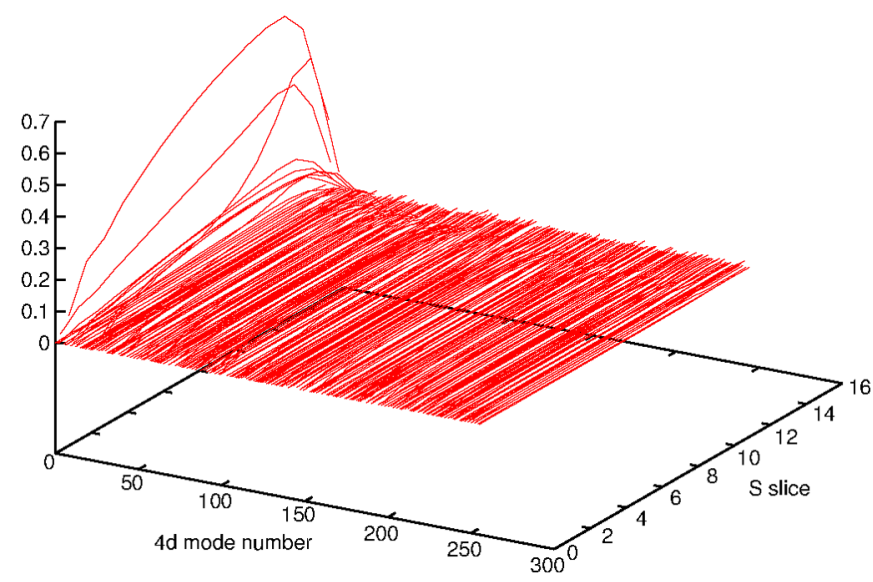

FIG. 10 (color online). Normalized overlap of different $s$ slices of a chiral five-dimensional eigenmode of $H_{\mathrm{DWF}}$ with the first 256 four-dimensional eigenmodes of $H_{W}$. We take the valence operator with $L_{s}=16$, and demonstrate numerically that only a few low-lying four-dimensional eigenmodes dominate the large $L_{s}$ contributions to chirality mixing operators such as $m_{\text {res }}$ by plotting the decomposition of the lowest five-dimensional mode $\left|\alpha_{0 j}(s)\right|^{2}$ as a function of the fifth coordinate $s$, and fourdimensional mode number $j$.

stant $H_{\mathrm{DWF}}$-eigenvector density [i.e. surfaces defined by $\Psi^{\dagger}(x, s) \Psi(x, s)=$ constant] obtained in our simulation. Examples of this are shown in Figs. 8 and 9, which represent lower-dimensional views, at different spatial locations, of the same eigenvector. A movie, which raster scans $x$ and $y$ as a unified "movie-time," can be obtained from [48].

Since the low modes of $H_{W}$ are scarce and very localized, it is to be expected that, if we are in a region of parameter space where their contribution is the dominant contribution to chiral symmetry breaking, quantities such as $m_{\text {res }}$ will display larger non-Gaussian tails (or "lumpiness") than if the exponentially falling contribution from extended states is dominant. Thus, one should not be surprised by the need for very high statistics to achieve a convincing estimate for $m_{\text {res }}$ in this region of parameter space.

\section{RESULTS FOR THE RESIDUAL MASS}

As discussed in Sec. IV, we calculate the residual mass from a ratio of correlation functions containing the axial Ward identity defect, $J_{5 q}^{b}$, defined in Eq. (A20):

$$
R(t)=\frac{\sum_{\vec{y}}\left\langle J_{5 q}^{b}(\vec{y}, t) P^{b}(0)\right\rangle}{\sum_{\vec{y}}\left\langle J_{5}^{b}(\vec{y}, t) P^{b}(0)\right\rangle}
$$

where $b$ is a fixed flavor index, and $J_{5}^{b}(\vec{y}, t)$ is the local fourdimensional pseudoscalar density defined on the $s=0$ and $s=L_{s}-1$ walls. The operator $P^{b}(0)$ is either $J_{5}^{b}(\vec{y}, t)$ or a spatially smeared variant defined at $t=0$. The residual 


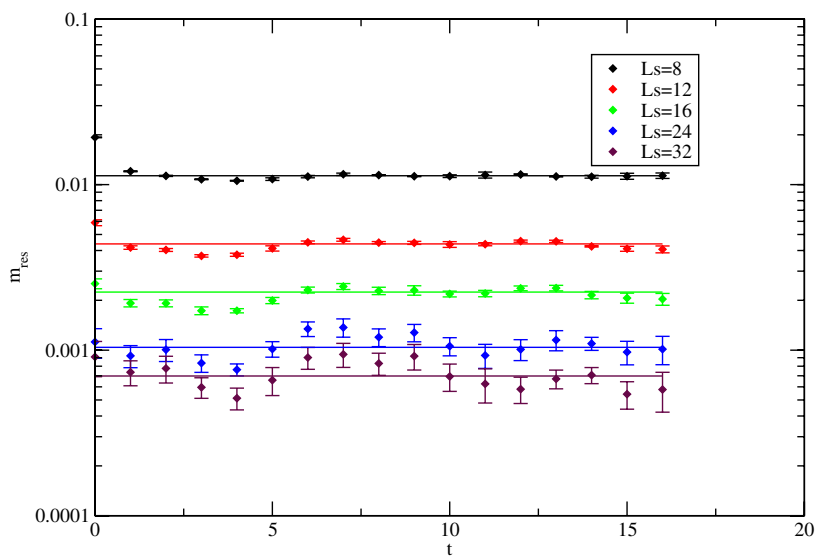

FIG. 11 (color online). Residual mass fits for valence quarks with $m=0.04$ and $L_{s}=8,12,16,24,32$ on the DBW2 $\beta=$ 0.72 ensemble with sea quark masses $m_{u d}=m_{s}=0.04$ and for the sea quark $L_{s}=8$.

mass is determined from a constant fit to $R(t)$ over a range of times, $t$, that are sufficiently large that lattice artifacts should be absent. Recall that the ratio $R(t)$ should equal the residual mass provided that the numerator and denominator of Eq. (34) are dominated by physical states. There is not a requirement that only a specific lowest state contributes. For the calculations reported here this average is performed over the range $7 \leq t \leq 16$. Figures $11-18$ contain plots of the fits of $R(t)$ to a constant. We use a combination of point, Coulomb gauge-fixed wall and Coulomb gauge-fixed exponential sources on the various data sets listed in Table II, and display the fitted values for $m_{\text {res }}$ in Tables III and IV for the DBW2 and Iwasaki gauge actions, respectively.

In order to conserve computer resources, we studied the dependence of $m_{\text {res }}$ on $L_{s}$ by working with our fixed set of gauge configurations generated with $L_{s}=8$ but varying the "valence" value of $L_{s}$ that appears in the propagators

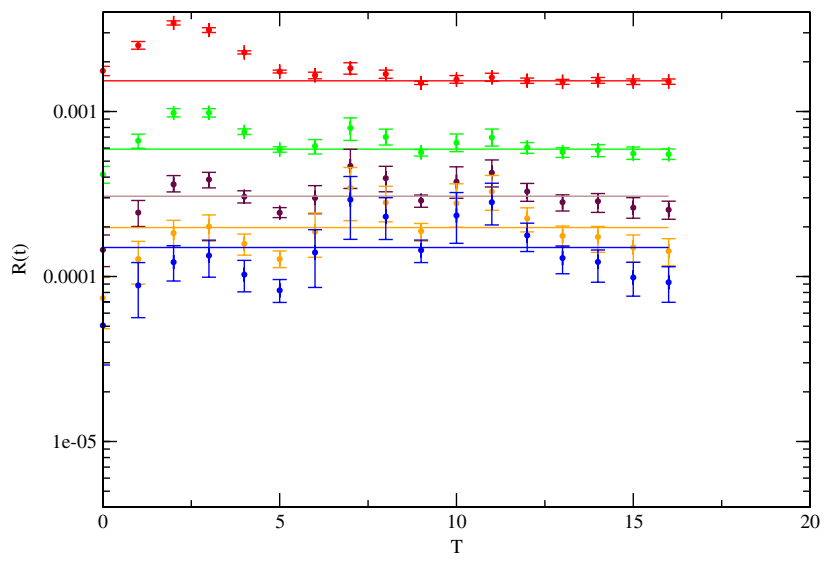

FIG. 12 (color online). Residual mass fits for valence quarks with $m=0.04$ and $L_{s}=8,12,16,24,32$ on the DBW2 $\beta=$ 0.764 ensemble with sea quark masses $m_{u d}=m_{s}=0.04$ and for the sea quark $L_{s}=8$.

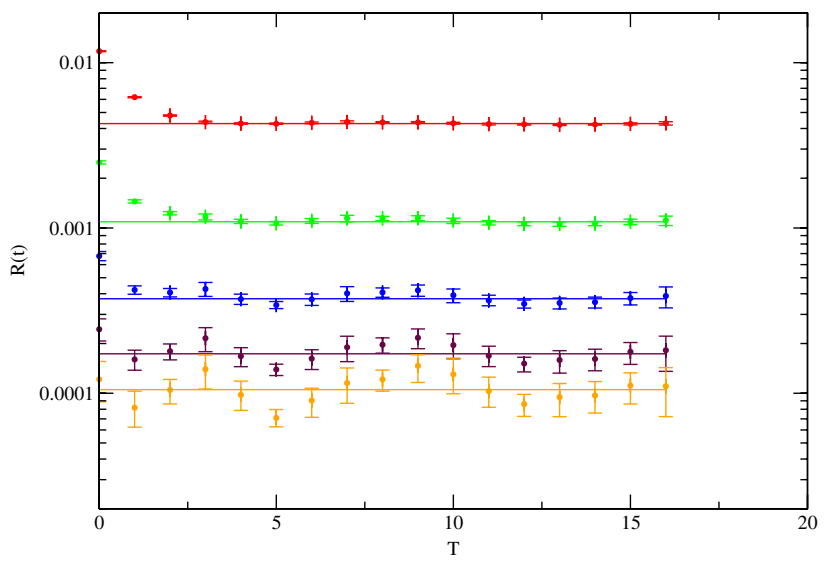

FIG. 13 (color online). Residual mass fits for valence quarks with $m=0.04$ and $L_{s}=8,12,16,24,32$ on the DBW2 $\beta=$ 0.78 ensemble with sea quark masses $m_{u d}=m_{s}=0.04$ and for the sea quark $L_{s}=8$.

used to compute the ratio in Eq. (34). The resulting $L_{s}$ dependence of the resulting $m_{\text {res }}$ can be viewed as a (likely good) approximation to what would have been obtained if we also varied the $L_{s}$ appearing in the fermion determinant. This also can be viewed as a self-consistent study of the properties of the five-dimensional transfer matrix defined on gauge configurations generated with this fixed, $L_{s}=8$ fermion action.

Next we examine the degree to which Eq. (14) describes the dependence of $m_{\text {res }}$ on $L_{s}$ in our simulations. Here we rewrite that equation in the form

$$
m_{\mathrm{res}}\left(L_{s}\right) \simeq\left(c_{1} e^{-\lambda_{c} L_{s}}+c_{2}\right) \frac{1}{L_{s}} .
$$

Recall that the first term is expected to come from extended states with eigenvalues $\lambda$ near the mobility edge: $\lambda_{c} \leq$ $\lambda \leq \lambda_{c}+\frac{1}{L_{s}}$. This term is exponentially suppressed for

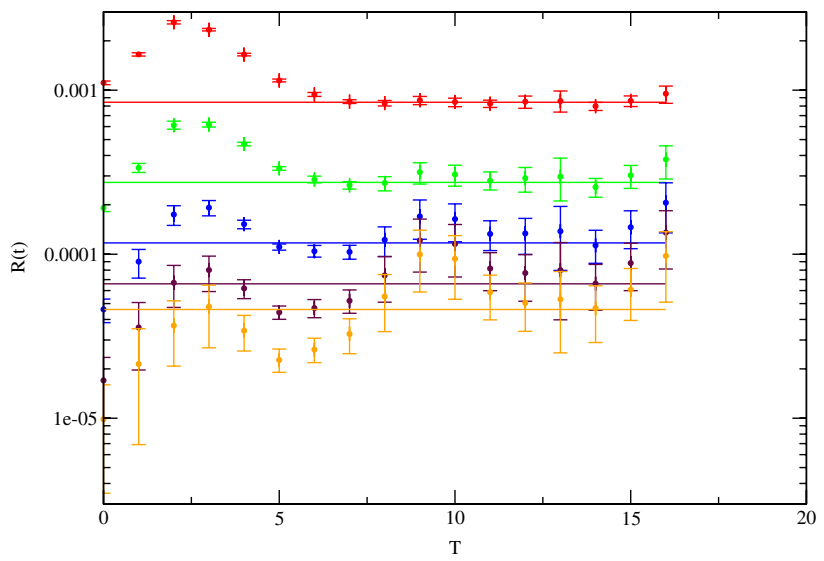

FIG. 14 (color online). Residual mass fits for valence quarks with $m=0.04$ and $L_{s}=8,12,16,24,32$ on the DBW2 $\beta=$ 0.80 ensemble with sea quark masses $m_{u d}=m_{s}=0.04$ and for the sea quark $L_{s}=8$. 
DAVID J. ANTONIO et al.



FIG. 15 (color online). Residual mass fits for valence quarks with $m=0.04$ and $L_{s}=8,12,16,24,32$ on the DBW2 $\beta=$ 0.88 ensemble with sea quark masses $m_{u d}=m_{s}=0.04$ and for the sea quark $L_{s}=8$.

large $L_{s}$ and thus can be easily reduced by increasing $L_{s}$. The second term arises from low-lying localized states with $0 \leq \lambda \leq \frac{1}{L_{s}}$. Here $c_{2}$ is proportional to the density of near-zero modes, $\rho(0)$. We require a demonstrably nonzero mobility edge $\lambda_{c}$ for safe QCD simulations with chiral formulations. It is acceptable to have a significant nonexponential component in $m_{\text {res }}$. However, the presence of such a term makes it difficult to significantly suppress chiral symmetry breaking effects by simply increasing $L_{s}$.

We show fits to the functional form in Eq. (35) for degenerate three flavor ensembles at several gauge couplings for the Iwasaki and DBW2 gauge actions in Figs. 19 and 20. The fitted parameters are listed in Table V. While the exponent $\lambda_{c}$ increases only slowly, the parameter $c_{2}$ falls by orders of magnitude as the lattice spacing decreases. Interpolating between the values of lattice spacing given in these tables suggests the localized near-zero

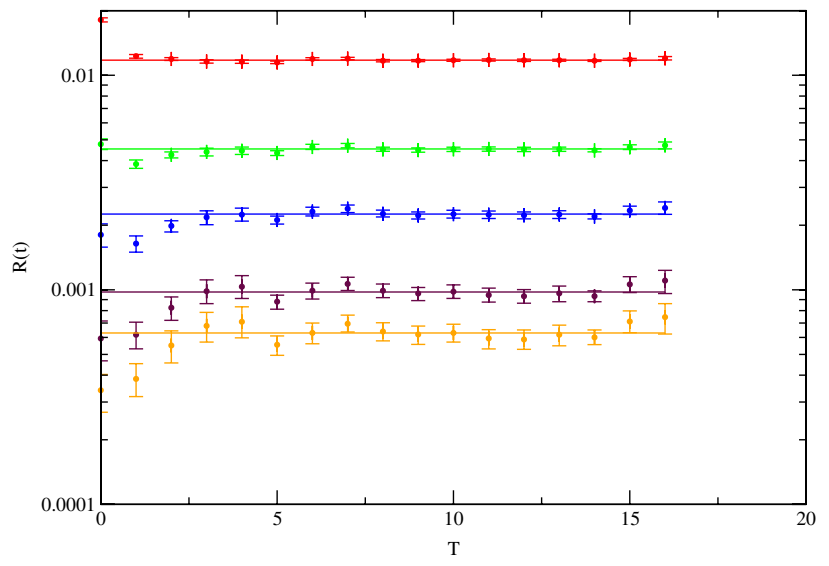

FIG. 16 (color online). Residual mass fits for valence quarks with $m=0.04$ and $L_{s}=8,12,16,24,32$ on the Iwasaki $\beta=$ 2.13 ensemble with sea quark masses $m_{u d}=m_{s}=0.04$ and for the sea quark $L_{s}=8$.
PHYSICAL REVIEW D 77, 014509 (2008)



FIG. 17 (color online). Residual mass fits for valence quarks with $m=0.04$ and $L_{s}=8,12,16,24,32$ on the Iwasaki $\beta=$ 2.2 ensemble with sea quark masses $m_{u d}=m_{s}=0.04$ and for the sea quark $L_{s}=8$.

modes are suppressed more by the DBW2 than the Iwasaki gauge action. This behavior will be correlated with the rate of topology change discussed in the next section.

We note that, while the residual mass term may be incorporated in a renormalized quark mass, one should extrapolate to $L_{s}=\infty$ to remove the residual chiral symmetry breaking effects of the $c_{5}$ term in Eq. (3). However, even for finite $L_{s}$, such terms are expected to be very small compared with the errors obtainable with nonperturbative improvement techniques for standard clover fermions, and thus can be treated as zero. Experience from clover fermions suggests that this level of error in the coefficient $c_{5}$ will certainly leave the results unmodified in practice, and that the unsuppressed $O\left(a^{2}\right)$ errors will be dominant in a continuum extrapolation. Figure 21 displays the dependence of the simplest of observables, the plaquette, on $L_{s}$ for the sea

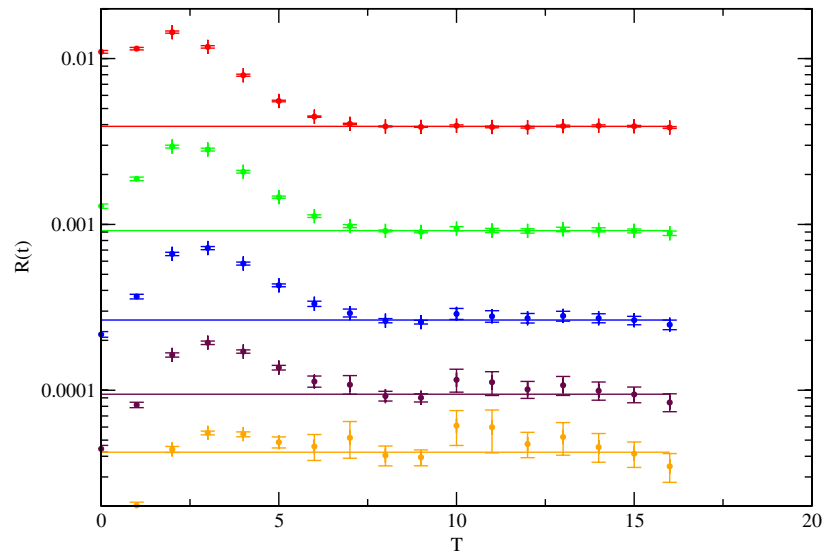

FIG. 18 (color online). Residual mass fits for valence quarks with $m=0.04$ and $L_{s}=8,12,16,24,32$ on the Iwasaki $\beta=$ 2.3 ensemble with sea quark masses $m_{u d}=m_{s}=0.04$ and for the sea quark $L_{s}=8$. 
TABLE II. Correlation functions generated for valence quarks with different extents of the fifth dimension on each of our $L_{s}=8$ dynamical ensembles. The valence and sea quark masses were $m_{u d}=m_{s}=0.04$. We denote point source correlators as "P," wall source correlators as "W," and exponentially smeared correlators as "S."

\begin{tabular}{l|ccccccc}
\hline \hline Data set & $L_{s}=8$ & $L_{s}=12$ & $L_{s}=16$ & $L_{s}=20$ & $L_{s}=24$ & $L_{s}=28$ & $L_{s}=32$ \\
\hline Iwasaki $\beta=2.13$ & $\mathrm{~S}$ & $\mathrm{~S}$ & $\mathrm{~S}$ & $\mathrm{~S}$ & $\mathrm{~S}$ & $\ldots$ & $\mathrm{S}$ \\
Iwasaki $\beta=2.2$ & $\mathrm{~W}, \mathrm{P}$ & $\mathrm{P}$ & $\mathrm{P}$ & $\mathrm{P}$ & $\mathrm{P}$ & $\ldots$ & $\mathrm{P}$ \\
Iwasaki $\beta=2.3$ & $\mathrm{~W}, \mathrm{P}$ & $\mathrm{P}$ & $\mathrm{P}$ & $\mathrm{P}$ & $\mathrm{P}$ & $\ldots$ & $\mathrm{P}$ \\
DBW2 $\beta=0.72$ & $\mathrm{~W}$ & $\mathrm{~W}$ & $\mathrm{~W}$ & $\cdots$ & $\mathrm{W}$ & $\ldots$ & $\mathrm{W}$ \\
DBW2 $\beta=0.764$ & $\mathrm{~W}$ & $\mathrm{P}$ & $\mathrm{P}$ & $\mathrm{P}$ & $\mathrm{P}$ & $\mathrm{P}$ & $\mathrm{P}$ \\
DBW2 $\beta=0.78$ & $\mathrm{~W}$ & $\mathrm{~W}, \mathrm{P}$ & $\mathrm{W}, \mathrm{P}$ & $\mathrm{W}, \mathrm{P}$ & $\mathrm{W}, \mathrm{P}$ & $\mathrm{P}$ & $\mathrm{P}$ \\
DBW2 $\beta=0.80$ & $\mathrm{~W}$ & $\mathrm{P}$ & $\mathrm{P}$ & $\mathrm{P}$ & $\mathrm{P}$ & $\mathrm{P}$ & $\mathrm{P}$ \\
DBW2 $\beta=0.88$ & $\mathrm{P}$ & $\mathrm{W}, \mathrm{P}$ & $\mathrm{W}, \mathrm{P}$ & $\mathrm{W}, \mathrm{P}$ & $\mathrm{W}, \mathrm{P}$ & $\cdots$ & $\mathrm{W}, \mathrm{P}$ \\
\hline \hline
\end{tabular}

TABLE III. Fitted residual mass values for valence quarks with different extents of the fifth dimension for the DBW2 gauge action on each of our $L_{s}=8$ dynamical ensembles. The valence and sea quark masses were $m_{u d}=m_{s}=0.04$.

\begin{tabular}{|c|c|c|c|c|c|c|c|}
\hline Data set & $L_{s}=8$ & $L_{s}=12$ & $L_{s}=16$ & $L_{s}=20$ & $L_{s}=24$ & $L_{s}=28$ & $L_{s}=32$ \\
\hline$\beta$ & $1.13(1) \times 10^{-2}$ & $4.4(1) \times 10^{-3}$ & $2.2(1) \times 10^{-3}$ & & $1.04(5) \times 10^{-3}$ & & $7.0(5) \times 10^{-4}$ \\
\hline$\beta=0.764$ & $5.46(4) \times 10^{-3}$ & $1.53(3) \times 10^{-3}$ & $5.9(2) \times 10^{-4}$ & $3.0(2) \times 10^{-4}$ & $2.0(2) \times 10^{-4}$ & $1.5(2) \times 10^{-4}$ & $1.2(2) \times 10^{-4}$ \\
\hline$\beta=0.78$ & $4.28(3) \times 10^{-3}$ & $1.09(2) \times 10^{-3}$ & $3.7(2) \times 10^{-4}$ & $1.7(1) \times 10^{-4}$ & $1.0(2) \times 10^{-4}$ & $7(1) \times 10^{-5}$ & $6(1) \times 10^{-5}$ \\
\hline$\beta=0.80$ & $3.39(2) \times 10^{-3}$ & $8.4(2) \times 10^{-4}$ & $2.7(1) \times 10^{-4}$ & $1.2(1) \times 10^{-4}$ & $6.6(8) \times 10^{-5}$ & $4.6(7) \times 10^{-5}$ & $3.6(7) \times 10^{-5}$ \\
\hline$\beta=0.88$ & $1.441(4) \times 10^{-3}$ & $2.40(2) \times 10^{-4}$ & $4.87(6) \times 10^{-5}$ & $1.14(3) \times 10^{-5}$ & $3.0(1) \times 10^{-6}$ & $\ldots$ & $3.3(4) \times 10^{-7}$ \\
\hline
\end{tabular}

TABLE IV. Fitted residual mass values for valence quarks with different extents of the fifth dimension for the Iwasaki gauge action on each of our $L_{s}=8$ dynamical ensembles. The valence and sea quark masses were $m_{u d}=m_{s}=0.04$.

\begin{tabular}{l|ccrrrr}
\hline \hline Data set & $L_{s}=8$ & $L_{s}=12$ & \multicolumn{1}{c}{$L_{s}=16$} & \multicolumn{1}{c}{$L_{s}=20$} & $L_{s}=24$ & $L_{s}=32$ \\
\hline$\beta=2.13$ & $1.18(1) \times 10^{-2}$ & $4.53(5) \times 10^{-3}$ & $2.25(5) \times 10^{-3}$ & $1.38(4) \times 10^{-3}$ & $9.8(4) \times 10^{-4}$ & $6.3(3) \times 10^{-4}$ \\
$\beta=2.2$ & $7.19(6) \times 10^{-3}$ & $2.25(4) \times 10^{-3}$ & $9.0(3) \times 10^{-4}$ & $4.6(2) \times 10^{-4}$ & $2.9(2) \times 10^{-4}$ & $1.7(2) \times 10^{-4}$ \\
$\beta=2.3$ & $3.90(2) \times 10^{-3}$ & $9.2(1) \times 10^{-4}$ & $2.65(6) \times 10^{-4}$ & $9.5(5) \times 10^{-5}$ & $4.2(4) \times 10^{-5}$ & $2.2(4) \times 10^{-5}$ \\
\hline \hline
\end{tabular}

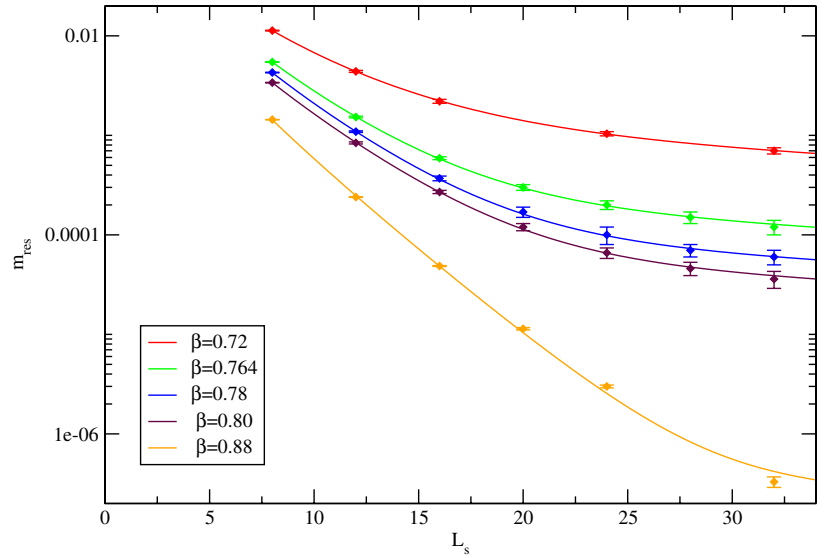

FIG. 19 (color online). Valence $m_{\text {res }}\left(L_{s}\right)$ dependence of partially quenched DWF with the DBW2 gauge action as a function of $L_{s}$, using $L_{s}=8$ and the indicated $\beta$ values on a $16^{3} \times 32$ volume for the ensembles. The sea and valence quark masses are 0.04 throughout.



FIG. 20 (color online). Valence $m_{\text {res }}\left(L_{s}\right)$ dependence of partially quenched DWF with the Iwasaki gauge action as a function of $L_{s}$, using $L_{s}=8$ and the indicated $\beta$ values on a $16^{3} \times 32$ volume for the ensembles. The sea and valence quark masses are 0.04 throughout. 
DAVID J. ANTONIO et al.

TABLE V. Fit parameters for the dependence of $m_{\text {res }}$ on $L_{s}$ for the Iwasaki and DBW2 gauge actions. We also give the lattice spacing obtained from the static potential [44,49] on the dynamical background for $m_{u d}=m_{s}=0.04$ without chiral extrapolation. This differs from the lattice spacing defined in the chiral limit presented in [44], and we only provide the lattice spacings here for illustration.

\begin{tabular}{l|l|cccc}
\hline \hline Action & \multicolumn{1}{|c|}{$\beta$} & $c_{1}$ & $\lambda_{c}$ & $c_{2}$ & $a^{-1}(\mathrm{GeV})$ \\
\hline Iwasaki & 2.13 & $0.344(6)$ & $0.191(2)$ & $0.0198(3)$ & $1.62(2)$ \\
Iwasaki & 2.2 & $0.301(4)$ & $0.219(2)$ & $0.0054(1)$ & $1.89(2)$ \\
Iwasaki & 2.3 & $0.262(3)$ & $0.268(2)$ & $0.0006(1)$ & $2.26(7)$ \\
DBW2 & 0.72 & $0.342(10)$ & $0.201(4)$ & $0.0220(4)$ & $1.40(4)$ \\
DBW2 & 0.764 & $0.298(6)$ & $0.252(3)$ & $0.0040(1)$ & $1.74(3)$ \\
DBW2 & 0.78 & $0.267(5)$ & $0.264(2)$ & $0.00188(7)$ & $1.82(2)$ \\
DBW2 & 0.80 & $0.216(5)$ & $0.265(3)$ & $0.00120(5)$ & $1.98(4)$ \\
DBW2 & 0.88 & $0.171(7)$ & $0.338(5)$ & $1.0(5) \times 10^{-5}$ & $\cdots$ \\
\hline \hline
\end{tabular}

quark content. It can be seen to be very weak (particularly for $L_{s} \geq 16$ ) and consistent with an exponential approach to the $L_{s} \rightarrow \infty$ limit, and is further evidence that all corrections to this limit are exponentially small.

\section{LOCALIZED MODES AND TOPOLOGY CHANGE}

Topology change, as defined by the index of an overlap operator, will be accompanied by a change of sign of a mode of $H_{W}\left(-M_{5}\right)$ in the region $-2<-M_{5}<0$. There will be an instant $\tau=\tau_{0}\left(M_{5}\right)$ in the molecular dynamics (MD) time at which $H_{W}\left(-M_{5}\right)$ has an exact zero mode for any appropriate choice of $M_{5}$. Such a level crossing leaves the topological index of an overlap operator based on this

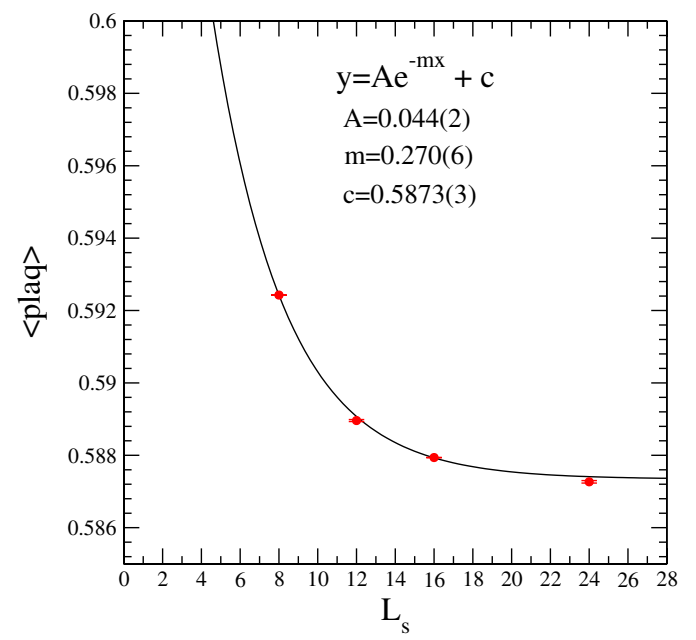

FIG. 21 (color online). Dependence of the plaquette as a function of the dynamical $L_{s}$, obtained from low statistics runs. The dependence of this observable shows the expected $C+$ $e^{-\alpha L_{s}}$ for an exponential approach to $L_{s}=\infty$, and thus demonstrates nicely the connection between finite $L_{s}$ DWF simulations and those with exactly chiral fermions.
PHYSICAL REVIEW D 77, 014509 (2008)

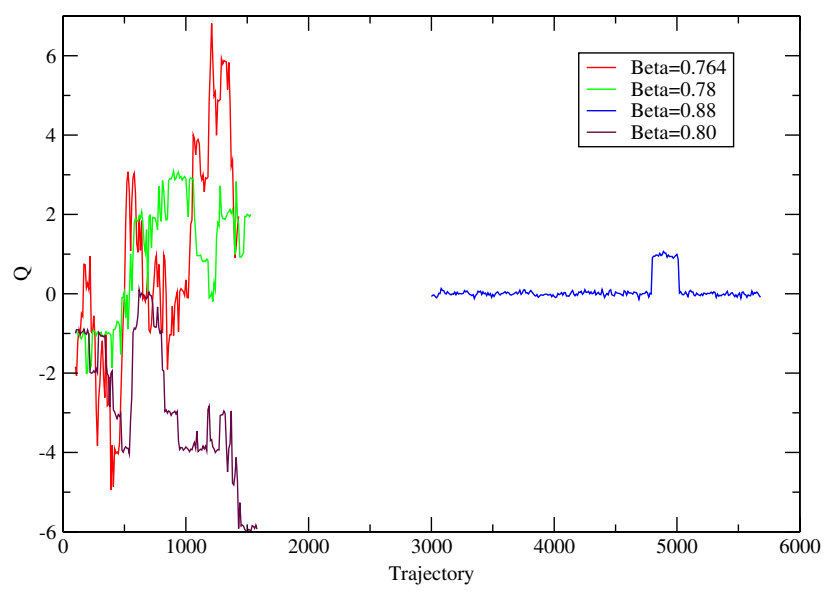

FIG. 22 (color online). The Monte Carlo trajectory history for global topological charge with the DBW2 gauge action at couplings $\beta=0.72,0.764,0.78,0.8$. The $\beta=0.88$ history is offset by 3000 and displays a clear loss of topology tunneling. With any reasonable computational effort, a simulation with the current algorithm and these parameters would be practically nonergodic.

kernel indefinite, depending on whether $-M_{5}$ is placed to the left or right of the level crossing, and it is reasonable to conjecture that low modes of $H_{W}\left(-M_{5}\right)$ play a significant role in topology change in dynamical simulations with current algorithms.

In this section we shall present results for the topological charge time history in our ensembles, and consider the relationship between tunneling rates, the eigenmode spectrum, and details of the algorithm and action.

In Figs. 22 and 23 we display the topological charge time histories for all our data sets measured using the same

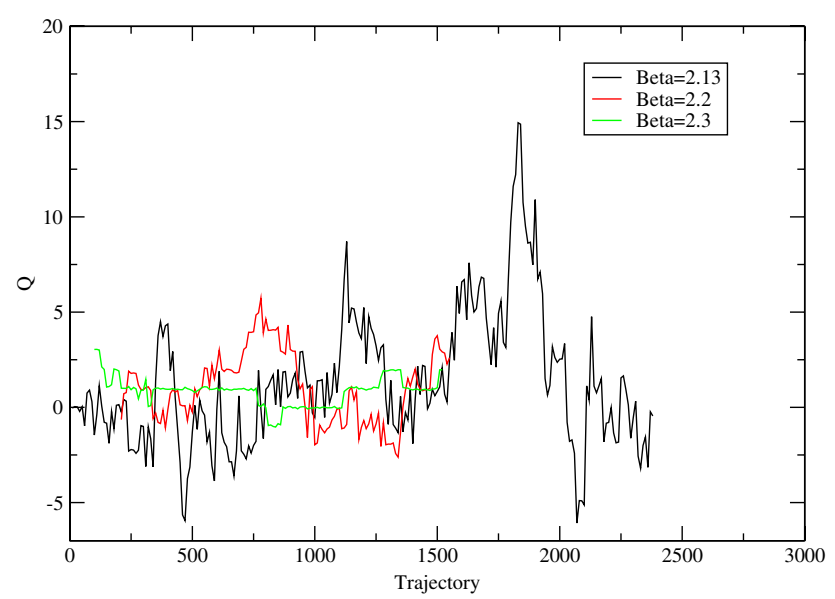

FIG. 23 (color online). The Monte Carlo trajectory history for global topological charge with the Iwasaki gauge action at couplings $\beta=2.13,2.2,2.3$. The tunneling rate remains acceptable and leaves headroom for taking a continuum limit with acceptable topological sampling without any further algorithmic improvement. 
$O\left(a^{2}\right)$-improved definition of the topological charge density as in Refs. [44,50], implemented using the Columbia physics system. It is certainly the case that the tunneling rate is substantially lower in those data sets with lower spectral densities. These are also the configurations with the finest lattice spacings.

Sampling all topological sectors has proven problematic with renormalization group improved gauge actions near the continuum limit, even in the quenched approximation; some authors previously found that the DBW2 gauge action samples topological sectors rather poorly at $a^{-1}=$ $3 \mathrm{GeV}$ in the quenched case [33], and this loss of tunneling is likely driven by the gauge action. Going nearer to the continuum limit yields an increasing potential barrier between topological sectors, arising from the gauge action, which prevents topology change in those algorithms that either use a local update, or, even worse, Hamiltonian evolution to propose a next configuration.

Whenever the determinant of $H_{W}$ does not directly enter the probability weight, the spectral density of low modes of $H_{W}$ found for the resulting gauge configurations is principally determined by the degree of roughness admitted by the gauge action, likely corresponding to fluxonlike configurations [51,52]. We conjecture that for domain wall fermions at practical values for $L_{s}$ the tunneling rate is controlled by this low mode density.

\section{A. Tunneling with dynamical overlap fermions}

Additional problems have been seen with the introduction of exactly chiral fermions in dynamical simulations. Exact implementations of the overlap operator introduce a discontinuous step in the action when a low eigenvalue of $H_{W}$ changes sign (and hence when the topological index of the configuration changes). This infinitely narrow step in the action is guaranteed to be unresolved by numerical integration for any nonzero time step, and leads to a high probability of rejection of the proposed configuration. The reflection/refraction algorithm $[53,54]$ is one sensible, but expensive, response to this.

Another response has been to develop actions which suppress low modes of $H_{W}$ with pseudofermion determinant estimation [55-57], and so suppress topology change. While many hadronic quantities are not likely to be sensitive to global topology (especially in the limit of large volume), there remains a serious concern that a modification which suppresses the change of global topology may also interfere with the creation of a proper distribution of local topological fluctuations. In the language of instantons, one should demonstrate that such an algorithm leads to a physical density of instanton-anti-instanton pairs-a quantity that may be important for QCD even when the global topology is fixed. However, we have poor tools for knowing whether this globally nonergodic algorithm is correctly sampling local topological fluctuations, given that we have thrown away our best diagnostic (and indeed the indicator that originally flagged the problem), i.e. global topology change.

So far with DWF we have obtained acceptable tunneling rates, and this is likely because the response to the lowest eigenvalues of the tanh approximation to the sign function, Eq. (1), is spread out at the level of our integration time step. More specifically, the determinant whose value is estimated contains a contribution $\tanh L_{s} \tanh ^{-1} \lambda$, in place of $\operatorname{sgn} \lambda$, and the transition has a width of $O\left(\frac{1}{L_{s}}\right)$. If we consider the low mode to be evolving in molecular dynamics time, with time step $\delta \tau$ and at a rate $\dot{\lambda}$, then the molecular dynamics integral will be accurate, provided

$$
\delta \tau \ll \frac{1}{L_{s} \dot{\lambda}} .
$$

There will be no practical impact on the tunneling rate from increasing $L_{s}$ while the condition Eq. (36) is held, and the problem may, in fact, never arise for any practical DWF simulation. In practice, the tunneling rate is determined by other effects, such as the low mode density determined by roughness admitted by the gauge action. We have also found a significant improvement in the tunneling rate, subsequent to this simulation arising from improving the quality of our stochastic estimate of the fermion force [58], where the use of a single stochastic estimate for the ratio of Pauli-Villars and light mass determinants was found to allow increased molecular dynamics step size and to increase the tunneling rate.

This also suggests a third possible response to this problem that arises in simulations with better approximations to the overlap operator than our DWF simulations: that is, to relax the approximation in the molecular dynamics evolution such that Eq. (36) is satisfied, while keeping the approximation accurate for the accept/reject step. This differential treatment of Metropolis and molecular dynamics steps is not possible with DWF simulations due to the fully five-dimensional pseudofermion fields, but it is possible for many approaches to the overlap operator.

\section{CONCLUSION}

When constrained to keep $L_{s}$ affordable, there is some degree of trade-off between the exactness of chirality and the thoroughness of topological sampling with domain wall fermions. Both are determined by the density of modes in the region where the tanh approximation is inaccurate, and this density is controlled by either the choice of the gauge action or the inclusion of additional unphysical terms in the action.

One might pause for a moment to ponder where to compromise. Using computer precision, (e.g. single precision) as the standard for achieving chiral symmetry invokes computer technology rather than physics for an answer to this important question. The authors' conclusion from this 
detailed study is that we should require adequate chirality, and by that we mean that the average symmetry breaking should not compromise the attractive simplification of electroweak current structure and off-shell $O(a)$ improvement for renormalization.

We have demonstrated in this paper that we can obtain $m_{\text {res }} \simeq O\left(10^{-3}\right)$ with both the DBW2 and Iwasaki gauge actions in the region of $a^{-1} \simeq 1.6 \mathrm{GeV}$. We have observed that dynamical simulations with the DBW2 gauge action rapidly lose topological tunneling as $\beta$ is increased. In contrast, headroom is left for going to weaker coupling, while maintaining good topological sampling, with the Iwasaki gauge action. This approach is certainly, at best, a stay of execution, as topological tunneling will be lost on sufficiently fine lattices. Such fine lattices are inaccessible with our current computers, and our conclusion is thus clear-the RBC and UKQCD collaborations are running [58] dynamical domain wall fermions with the Iwasaki gauge action and $L_{s} \geq 16$. We started from $a^{-1} \simeq$ $1.6 \mathrm{GeV}$, and thus we are able to maintain both excellent topological sampling and acceptable chiral symmetry violation.

\section{ACKNOWLEDGMENTS}

We thank Dong Chen, Calin Cristian, Zhihua Dong, Alan Gara, Andrew Jackson, Changhoan Kim, Ludmila Levkova, Xiaodong Liao, Guofeng Liu, Konstantin Petrov, and Tilo Wettig for developing with us the QCDOC machine and its software. This development and the resulting computer equipment used in this calculation were funded by the U.S. DOE Grant No. DE-FG0292ER40699, PPARC JIF Grant No. PPA/J/S/1998/00756 and by RIKEN. This work was supported by DOE Grant No. DE-FG02-92ER40699 and No. DE-AC0298CH10886 and PPARC Grant No. PPA/G/O/2002/ 00465, No. PP/D000238/1, and No. PP/C504386/1. A. H. is supported by the UK Royal Society. We thank BNL, EPCC, RIKEN, and the U.S. DOE for supporting the computing facilities essential for the completion of this work.

\section{APPENDIX: DOMAIN WALL FERMION TRANSFER MATRIX}

Here we collect some useful formulas that describe the transfer matrix formalism as applied to domain wall fermions by Furman and Shamir [3]. While we do not present a derivation of these formulas, they follow reasonably directly from the results presented by Furman and Shamir, Narayanan and Neuberger [59], and Lüscher [60].

We begin with the five-dimensional domain wall fermion Dirac operator defined in Eqs. (5)-(7). (This definition of the domain wall fermion operator is consistent with our earlier papers, but differs by a Hermitian conjugate from the original notation of Furman and Shamir.) We also identify two important four-dimensional operators which play a central role in Lüscher's original transfer matrix construction [60]:

$$
\begin{gathered}
B_{x, x^{\prime}}=\left(5-M_{5}\right) \delta_{x, x^{\prime}}-\frac{1}{2} \sum_{\mu}\left[\delta_{x+\mu, x^{\prime}} U_{x, \mu}+\delta_{x, x^{\prime}+\mu} U_{x^{\prime}, \mu}^{\dagger}\right], \\
C_{x, x^{\prime}}=\frac{1}{2} \sum_{\mu}\left[\delta_{x+\mu, x^{\prime}} U_{x, \mu}-\delta_{x, x^{\prime}+\mu} U_{x^{\prime}, \mu}^{\dagger}\right] \sigma^{\mu} .
\end{gathered}
$$

Here we have adopted a spinor basis in which $\gamma^{5}$ is diagonal. In this basis the $\gamma$ matrices can be written

$$
\gamma^{\mu}=\left(\begin{array}{cc}
0 & \sigma^{\mu} \\
\left(\sigma^{\mu}\right)^{\dagger} & 0
\end{array}\right), \quad \gamma^{5}=\left(\begin{array}{cc}
1 & 0 \\
0 & -1
\end{array}\right),
$$

and the four $2 \times 2$ matrices $\sigma^{\mu}$ appearing in both Eqs. (A2) and (A3) are given by $\sigma^{\mu}=(i, \vec{\sigma})$, where $\vec{\sigma}$ represents the three standard Pauli matrices and $\frac{1}{2}\left(1 \pm \gamma^{5}\right)$ projects onto right-/left-handed states. Thus, the matrices $B$ and $C$ have a $2 \times 2$-dimensional spin structure and appear directly in the matrix $D_{x, x^{\prime}}^{\|}\left(M_{5}\right)$ of Eq. (6):

$$
D_{x, x^{\prime}}^{\|}\left(M_{5}\right)=I-\left(\begin{array}{cc}
B & C \\
C^{\dagger} & B
\end{array}\right)
$$

Next we need to introduce three pairs of related fermion fields. The first two pairs are the five-dimensional Grassmann variables $\Psi_{x, s}$ and $\bar{\Psi}_{x, s}$ and the corresponding Fock-space operators $\hat{\psi}_{x}$ and $\hat{\bar{\psi}}_{x}$. The field operators $\hat{\psi}_{x}$ and $\hat{\bar{\psi}}_{x}$ can be written in terms of two-component field operators $\hat{c}_{x}$ and $\hat{d}_{x}$ and their Hermitian conjugates:

$$
\begin{gathered}
\hat{\psi}=\left(\begin{array}{c}
B^{-(1 / 2)}\left(\hat{d}^{\dagger}\right)^{t} \\
B^{-(1 / 2)} \hat{c}
\end{array}\right), \\
\hat{\bar{\psi}}=\left(-\hat{d}^{t} B^{-(1 / 2)}, \hat{c}^{\dagger} B^{-(1 / 2)}\right) .
\end{gathered}
$$

Here the operators $\hat{c}$ and $\hat{d}$ both act on vectors in the Fock space and carry space-time, color, and spinor indices. As Fock-space operators, they can be thought of as

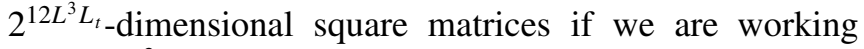
with a $L^{3} \times L_{t}$ space-time lattice. We treat their spacetime, color, and spin indices as the index on a $6 L^{3} L_{t} \times 1$ column vector. It is on this vector that the $6 L^{3} L_{t} \times 6 L^{3} L_{t}$ matrix $B^{-(1 / 2)}$ acts in Eqs. (A5) and (A6). We use the conventions that the Hermitian conjugate, represented by the superscript $\dagger$, takes the Hermitian conjugate of both the Fock-space operator and the space-time-flavor-spin matrix. The transpose, represented by the superscript $t$, acts only on the space-time-flavor-spin matrix.

Finally we introduce two further operators:

$$
\begin{gathered}
\hat{R}(\hat{c}, \hat{d})=\exp \left\{\hat{d}^{t}\left(B^{-(1 / 2)} C B^{-(1 / 2)}\right) \hat{c}\right\}, \\
\hat{W}\left(\hat{c}^{\dagger}, \hat{d}^{\dagger}, \hat{c}, \hat{d}\right)=\exp \left\{-\hat{c}^{\dagger} \ln (B) \hat{c}-\hat{d}^{\dagger} \ln (B) \hat{d}\right\} .
\end{gathered}
$$


Using these operators we can then connect the Feynman path integral, which provides the original definition of domain wall fermion lattice theory, with a Fock-space operator expression. For simplicity, we consider an example of the five-dimensional fermion propagator, because a general fermionic Green's function follows exactly the same pattern:

$$
\begin{aligned}
& \int \prod_{x^{\prime}, s^{\prime}} d\left[\Psi_{x^{\prime}, s^{\prime}}\right] d\left[\bar{\Psi}_{x^{\prime}, s^{\prime}}\right] e^{\bar{\Psi} D_{\mathrm{DWF}} \Psi} \Psi_{x_{1}, s_{1}} \bar{\Psi}_{x_{2}, s_{2}} \\
& =\prod_{s=0}^{L_{s}-1}\left\{\prod _ { x } \int d \overline { \Psi } _ { x , s } d \Psi _ { x , s } \operatorname { e x p } \left[\bar{\Psi}_{s}^{R} \Psi_{s+1}^{L}+\bar{\Psi}_{s+1}^{L} \Psi_{s}^{R}\right.\right. \\
& \text { - } \bar{\Psi}_{s}^{L} B \Psi_{s}^{R}-\bar{\Psi}_{s}^{R} B \Psi_{s}^{L}-\bar{\Psi}_{s}^{R} C^{\dagger} \Psi_{s}^{R} \\
& \left.\left.+\bar{\Psi}_{s}^{L} C \Psi_{s}^{L}\right]\right\} \Psi_{x_{1}, s_{1}} \bar{\Psi}_{x_{2}, s_{2}} \\
& =(\operatorname{det} B)^{2 L_{s}} \operatorname{tr}\left\{\left(\hat{W}^{1 / 2} \hat{R} \hat{R}^{\dagger} \hat{W}^{1 / 2}\right)^{s_{1}} \hat{W}^{1 / 2} \hat{R} \hat{\psi}_{x_{1}} \hat{R}^{\dagger}\right. \\
& \times \hat{W}^{1 / 2}\left(\hat{W}^{1 / 2} \hat{R} \hat{R}^{\dagger} \hat{W}^{1 / 2}\right)^{s_{2}-s_{1}-1} \hat{W}^{1 / 2} \hat{R} \hat{\bar{\psi}}_{x_{2}} \hat{R}^{\dagger} \\
& \left.\times \hat{W}^{1 / 2}\left(\hat{W}^{1 / 2} \hat{R} \hat{R}^{\dagger} \hat{W}^{1 / 2}\right)^{L_{s}-s_{2}-1} \mathcal{O}\left(m_{f}\right)\right\},
\end{aligned}
$$

where

$$
\mathcal{O}\left(m_{f}\right)=\prod_{\alpha}\left\{\left(\hat{c}_{\alpha} \hat{c}_{\alpha}^{\dagger}+m_{f} \hat{c}_{\alpha}^{\dagger} \hat{c}_{\alpha}\right)\left(\hat{d}_{\alpha} \hat{d}_{\alpha}^{\dagger}+m_{f} \hat{d}_{\alpha}^{\dagger} \hat{d}_{\alpha}\right)\right\}
$$

and $\alpha$ represents space-time, spin, and color indices. While a complete derivation of the result in Eq. (A10) is beyond the scope of this paper, one of the intermediate steps is shown in Eq. (A9). In that equation the Grassmann fields $\Psi$ and $\bar{\Psi}$ have been expressed in terms of their chiral components according to

$$
\Psi=\left(\begin{array}{l}
\Psi^{R} \\
\Psi^{L}
\end{array}\right), \quad \bar{\Psi}=\left(\bar{\Psi}^{L} \bar{\Psi}^{R}\right) .
$$

A complete derivation of Eq. (A10) can be constructed from Refs. $[3,59,60]$. Note, the Grassmann fields $\Psi_{L_{s}}^{L}$ and $\bar{\Psi}_{L_{s}}^{L}$ appear in the integrand in Eq. (A9) but are not among the variables of integration. Instead, these fields are to be evaluated as $-m_{f}$ times the corresponding fields at $s=0$ : $-m_{f} \Psi_{s=0}^{L}$ and $-m_{f} \bar{\Psi}_{s=0}^{L}$, respectively.

Following Furman and Shamir, we identify the Fockspace transfer matrix $T$ as

$$
T=(\operatorname{det} B) \hat{W}^{1 / 2} \hat{R} \hat{R}^{\dagger} \hat{W}^{1 / 2}=e^{-\hat{a}^{\dagger} H_{T} \hat{a}}
$$

$$
\begin{gathered}
\text { where } \hat{a}=\left(\begin{array}{c}
\left(\hat{d}^{\dagger}\right)^{t} \\
(\hat{c})
\end{array}\right), \quad \hat{a}^{\dagger}=\left(\hat{d}^{t}, \hat{c}^{\dagger}\right), \quad \text { and } \\
e^{-H_{T}}=\left(\begin{array}{cc}
B+C B^{-1} C^{\dagger} & C B^{-1} \\
B^{-1} C^{\dagger} & B^{-1}
\end{array}\right) .
\end{gathered}
$$

The unusual arrangement of the operators $\hat{\psi}_{x_{1}}$ and $\hat{\bar{\psi}}_{x_{2}}^{\dagger}$ and two missing factors of $T$ in Eq. (A10) can be understood if, following Furman and Shamir, we recognize that the upper component of $\hat{\psi}$ and the lower component of $\hat{\bar{\psi}}$ commute with $\hat{R}^{\dagger}$, while the lower component of $\hat{\psi}$ and the upper component of $\hat{\bar{\psi}}$ commute with $\hat{R}$. This allows the operators $\hat{\psi}$ and $\hat{\bar{\psi}}$ to be extracted from between the factors $\hat{W}^{1 / 2} \hat{R}$ and $\hat{R}^{\dagger} \hat{W}^{1 / 2}$, so that the two missing factors of $T$ can be assembled. [Note, the operators $\hat{\psi}$ and $\hat{\bar{\psi}}$ do not commute with the operator $\hat{W}^{1 / 2}$, but instead have the nonlocal factor of $B^{-(1 / 2)}$ appearing in Eqs. (A5) and (A6) removed by the proposed interchange.]

We will demonstrate this rearrangement of operators by working out two examples. In the first, we translate the expectation value $\langle\bar{q}(x) q(x)\rangle$ (the chiral condensate) into this transfer matrix language. Recall that the physical, four-dimensional Grassmann fields are defined by

$$
\begin{aligned}
& q(x)=P_{L} \Psi_{x, 0}+P_{R} \Psi_{x, L_{s}-1}, \\
& \bar{q}(x)=\bar{\Psi}_{x, 0} P_{R}+\bar{\Psi}_{x, L_{s}-1} P_{L},
\end{aligned}
$$

where we have used $P_{R / L}=\left(1 \pm \gamma^{5}\right) / 2$. If these expressions are substituted into Eq. (A10), we obtain

$$
\begin{aligned}
& \langle\bar{q}(x) q(x)\rangle=-(\operatorname{det} B)^{L_{s}} \operatorname{tr}\left\{\hat{W}^{1 / 2} \hat{R}\left(P_{L} \hat{\psi}_{x}\right)_{\alpha} \hat{R}^{\dagger} \hat{W}^{1 / 2} T^{L_{s}-2}\right. \\
& \left.\times \hat{W}^{1 / 2} \hat{R}\left(\hat{\bar{\psi}}_{x} P_{L}\right)_{\alpha} \hat{R}^{\dagger} \hat{W}^{1 / 2} \mathcal{O}\left(m_{f}\right)\right\} \\
& +(\operatorname{det} B)^{L_{s}} \operatorname{tr}\left\{\hat{W}^{1 / 2} \hat{R}\left(\hat{\bar{\psi}} P_{R}\right)_{\alpha} \hat{R}^{\dagger} \hat{W}^{1 / 2} T^{L_{s}-2}\right. \\
& \left.\times \hat{W}^{1 / 2} \hat{R}\left(P_{R} \hat{\psi}_{x}\right)_{\alpha} \hat{R}^{\dagger} \hat{W}^{1 / 2} \mathcal{O}\left(m_{f}\right)\right\} \text {. }
\end{aligned}
$$

The final step involves commuting the $P_{L} \hat{\psi}$ and $\hat{\bar{\psi}} P_{R}$ past the factor $\hat{W}^{1 / 2} \hat{R}$ to stand on the far left. Similarly, the operators $P_{R} \hat{\psi}$ and $\hat{\bar{\psi}} P_{L}$ on the right side can be moved past the factor $\hat{R}^{\dagger} \hat{W}^{1 / 2}$ to stand directly to the left of the operator $\mathcal{O}\left(m_{f}\right)$. The resulting expression can be written simply in terms of the operators $\hat{a}$ and $\hat{a}^{\dagger}$ :

$$
\begin{aligned}
\langle\bar{q}(x) q(x)\rangle= & -(\operatorname{det} B)^{L_{s}} \operatorname{tr}\left\{\left[\left(P_{L} \hat{a}_{x}\right)_{\alpha} T^{L_{s}}\left(\hat{a}_{x}^{\dagger} P_{L}\right)_{\alpha}\right.\right. \\
& \left.\left.+\left(\hat{a}_{x}^{\dagger} P_{R}\right)_{\alpha} T^{L_{s}}\left(P_{R} \hat{a}_{x}\right)_{\alpha}\right] \mathcal{O}\left(m_{f}\right)\right\} .
\end{aligned}
$$

As a second example, consider the Green's function $\left\langle P_{L} q(x) J_{5 q}^{b}(z) \bar{q}(y) P_{L}\right\rangle$ containing the midpoint operator $J_{5 q}^{a}$ that occurs in the divergence of the five-dimensional flavor nonsinglet axial current introduced by Furman and Shamir [3]:

$$
\begin{aligned}
J_{5 q}^{b}(x)= & -\bar{\Psi}\left(x, L_{s} / 2-1\right) P_{L} t^{b} \Psi\left(x, L_{s} / 2\right) \\
& +\bar{\Psi}\left(x, L_{s} / 2\right) P_{R} t^{b} \Psi\left(x, L_{s} / 2-1\right) .
\end{aligned}
$$

Here $b$ is a flavor index and $t^{b}$ is a flavor generator.

Using Eq. (A10) for this product of four Grassmann fields we find 


$$
\begin{aligned}
& \left\langle P_{L} q(x) J_{5 q}^{b}(z) \bar{q}(y) P_{L}\right\rangle=-(\operatorname{det} B)^{L_{s}} \operatorname{tr}\left\{\hat { W } ^ { 1 / 2 } \hat { R } ( P _ { L } \hat { \psi } _ { x } ) \hat { R } ^ { \dagger } \hat { W } ^ { 1 / 2 } T ^ { L _ { s } / s - 2 } \left[-\hat{W}^{1 / 2} \hat{R}\left(\hat{\bar{\psi}}_{z} P_{L} t^{b}\right)_{\alpha} \hat{R}^{\dagger} \hat{W}^{1 / 2} \hat{W}^{1 / 2} \hat{R}\left(P_{L} \hat{\psi}_{z}\right)_{\alpha} \hat{R}^{\dagger} \hat{W}^{1 / 2}\right.\right. \\
& \left.\left.-\hat{W}^{1 / 2} \hat{R}\left(t^{b} P_{R} \hat{\psi}_{z}\right)_{\alpha} \hat{R}^{\dagger} \hat{W}^{1 / 2} \hat{W}^{1 / 2} \hat{R}\left(\hat{\bar{\psi}}_{z} P_{R}\right)_{\alpha} \hat{R}^{\dagger} \hat{W}^{1 / 2}\right] T^{L_{s} / s-2} \hat{W}^{1 / 2} \hat{R}\left(\hat{\bar{\psi}}_{y} P_{R}\right) \hat{R}^{\dagger} \hat{W}^{1 / 2} \mathcal{O}\left(m_{f}\right)\right\} \text {. }
\end{aligned}
$$

Performing the same operator manipulations as used to obtain Eq. (A18) we find

$$
\begin{aligned}
\left\langle P_{L} q(x) J_{5 q}^{b}(z) \bar{q}(y) P_{L}\right\rangle= & (\operatorname{det} B)^{L_{s}} \operatorname{tr}\left\{P_{L} \hat{a}_{x} T^{L_{s} / 2} \hat{a}_{z}^{\dagger} t^{b} \hat{a}_{z}\right. \\
& \left.\times T^{L_{s} / 2} \hat{a}_{y}^{\dagger} P_{L} \mathcal{O}\left(m_{f}\right)\right\} .
\end{aligned}
$$

Here the unit displacement in $s$ between the two factors in $J_{5 q}^{a}$ has disappeared, leaving a very symmetrical expression. The missing $\gamma^{5}$ matrix, which one would expect in an axial matrix element, has its origins in the minus sign that is present in Eq. (A6) but absent in Eq. (A14). This factor could be restored if we introduced $\hat{\bar{a}}_{x}=\hat{a}_{x}^{\dagger} \gamma^{5}$, a fivedimensional analogue of the role played by $\gamma^{0}$ in the standard treatment of the four-dimensional Dirac operator.

As a final topic for this appendix, we will examine the effects of the matrix $T^{L_{s}}$ in Eq. (A18). Again following Furman and Shamir, we introduce the eigenvectors and eigenvalues of the operator $H_{T}$ defined in Eq. (A15):

$$
\begin{array}{cc}
H_{T} \phi_{k^{+}}^{+}=E_{k^{+}}^{+} \phi_{k^{+}}^{+}, & 1 \leq k^{+} \leq N^{+}, \\
H_{T} \phi_{k^{-}}^{-}=-E_{k^{-}}^{-} \phi_{k^{-}}^{-}, & 1 \leq k^{-} \leq N^{-},
\end{array}
$$

where $E_{n}^{\mp} \geq 0$, and we write the operator $\hat{a}_{x}$ in terms of this basis, filling the Dirac sea in the usual way:

$$
\hat{a}_{x}=\sum_{k+=1}^{N^{+}} \phi_{k^{+}}^{+}(x) \hat{o}_{k}+\sum_{k^{-}=1}^{N^{-}} \phi_{k^{-}}^{-}(x) \hat{p}_{k^{-}}^{\dagger} .
$$

Let $\left|0_{H}\right\rangle$ be the Fock-space state $\left|0_{H}\right\rangle$ which is the eigenstate of $T$ with the largest eigenvalue. This state obeys

$$
\hat{o}_{k^{+}}\left|0_{H}\right\rangle=\hat{p}_{k^{-}}\left|0_{H}\right\rangle=0
$$

for all $k^{+}$and $k^{-}$and

$$
T\left|0_{H}\right\rangle=\lambda_{\max }\left|0_{H}\right\rangle=\exp \left\{\sum_{k^{-}=1}^{N^{-}} E_{k^{-}}^{-}\right\}\left|0_{H}\right\rangle .
$$

Finally we define the normalized operator $\hat{T}=T / \lambda_{\max }$ making unity the largest eigenvalue of $\hat{T}$ and $\left|0_{H}\right\rangle$ the corresponding eigenstate. The matrix $\hat{T}$ can be written

$$
\begin{gathered}
\hat{T}=T / \lambda_{\max }=\exp \left\{-\hat{a}^{\dagger} H \hat{a}\right\} / \lambda_{\max } \\
=\exp \left\{-\sum_{k^{+}=1}^{N^{+}} E_{k^{+}}^{+} \hat{o}_{k^{+}}^{\dagger} \hat{o}_{k^{+}}+\sum_{k^{-}=1}^{N^{-}} E_{k^{-}}^{-} \hat{p}_{k^{-}} \hat{p}_{k^{-}}^{\dagger}\right\} / \lambda_{\max }
\end{gathered}
$$

$$
=\exp \left\{-\sum_{k^{+}=1}^{N^{+}} E_{k^{+}}^{+} \hat{o}_{k^{+}}^{\dagger} \hat{o}_{k^{+}}-\sum_{k^{-}=1}^{N^{-}} E_{k^{-}}^{-} \hat{p}_{k^{-}}^{\dagger} \hat{p}_{k^{-}}\right\} .
$$

Thus, in the limit of large $L_{s}, \hat{T}^{L_{s}}$ becomes the projection operator onto the state $\left|0_{H}\right\rangle$, separating the right and left operators in an equation such as Eq. (A18) into two separate factors, linked by the operator $\mathcal{O}\left(m_{f}\right)$. If we further take the limit $m_{f}=0$ and define a surface vacuum state $\left|0_{S}\right\rangle$, which is annihilated by the operators $\hat{c}_{x}$ and $\hat{d}_{x}$,

$$
\hat{c}_{x}\left|0_{S}\right\rangle=\hat{d}_{x}\left|0_{S}\right\rangle=0 \text {, }
$$

then the operator $\mathcal{O}\left(m_{f}=0\right)$, given in Eq. (A11), reduces to the projection operator $\left|0_{S}\right\rangle\left\langle 0_{S}\right|$ and the left- and righthanded sectors become completely independent matrix elements between $\left|0_{S}\right\rangle$ and $\left|0_{H}\right\rangle$.

This can be summarized by evaluating a general Green's function depending on the four-dimensional fields $q$ and $\bar{q}$. This is most easily done if the fermion fields in the original Green's function are ordered with the left-handed chiral fields $\left(P_{L} q\right.$ and $\left.\bar{q} P_{R}\right)$ on the left and the right-handed chiral fields $\left(P_{R} q\right.$ and $\left.\bar{q} P_{L}\right)$ on the right:

$$
\begin{aligned}
& \frac{1}{\left(\operatorname{det} B \lambda_{\max }\right)^{L_{s}}}\left\langle\mathcal{O}_{L}\left[P_{L} q, \bar{q} P_{R}\right] \mathcal{O}_{R}\left[P_{R} q, \bar{q} P_{L}\right]\right\rangle_{L_{s}} \quad(\mathrm{~A} 32) \\
&= \frac{1}{\left(\lambda_{\max }\right)^{L_{s}}} \operatorname{tr}\left\{\mathcal{O}_{L}\left[P_{L} \hat{a},-\hat{a}^{\dagger} P_{R}\right] T^{L_{s}} \mathcal{O}_{R}\left[P_{R} \hat{a}, \hat{a}^{\dagger} P_{L}\right] \mathcal{O}\left(m_{f}\right)\right\} \\
& \underset{L_{s} \rightarrow \infty}{\rightarrow} \operatorname{tr}\left\{\mathcal{O}_{L}\left[P_{L} \hat{a},-\hat{a}^{\dagger} P_{R}\right]\left|0_{H}\right\rangle\left\langle 0_{H}\right| \mathcal{O}_{R}\left[P_{R} \hat{a}, \hat{a}^{\dagger} P_{L}\right] \mathcal{O}\left(m_{f}\right)\right\} \\
& \underset{m_{f} \rightarrow 0}{\rightarrow}\left\langle 0_{S}\left|\mathcal{O}_{L}\left[P_{L} \hat{a},-\hat{a}^{\dagger} P_{R}\right]\right| 0_{H}\right\rangle\left\langle 0_{H}\left|\mathcal{O}_{R}\left[P_{R} \hat{a}, \hat{a}^{\dagger} P_{L}\right]\right| 0_{S}\right\rangle,
\end{aligned}
$$

where the quantities $\mathcal{O}_{L}$ and $\mathcal{O}_{R}$ are two ordered polynomials in their two arguments.

Corrections to the limit $L_{s} \rightarrow \infty$ can be written by including eigenvectors of $\hat{T}$ with smaller eigenvalues. Such corrections can be easily obtained from Eq. (A30):

$$
\begin{aligned}
\hat{T}^{L_{s} \approx} & \left|0_{H}\right\rangle\left\langle 0_{H}\left|+\sum_{k^{+}=1}^{N^{+}} e^{-E_{k^{+}}^{+} L_{s}} \hat{o}_{k^{+}}^{\dagger}\right| 0_{H}\right\rangle\left\langle 0_{H}\right| \hat{o}_{k^{+}} \\
& +\sum_{k^{-}=1}^{N^{-}} e^{-E_{k^{-}}^{-} L_{s}} \hat{p}_{k^{-}}^{\dagger}\left|0_{H}\right\rangle\left\langle 0_{H}\right| \hat{p}_{k^{-}}+\ldots
\end{aligned}
$$


[1] D. B. Kaplan, Phys. Lett. B 288, 342 (1992).

[2] H. Neuberger, Phys. Lett. B 417, 141 (1998).

[3] V. Furman and Y. Shamir, Nucl. Phys. B439, 54 (1995).

[4] S. Aoki, Phys. Rev. D 30, 2653 (1984).

[5] M. A. Clark and A.D. Kennedy, Nucl. Phys. B, Proc. Suppl. 129, 850 (2004).

[6] M.A. Clark, A. D. Kennedy, and Z. Sroczynski, Nucl. Phys. B, Proc. Suppl. 140, 835 (2005).

[7] P. Boyle et al., Nucl. Phys. B, Proc. Suppl. 140, 169 (2005).

[8] P. A. Boyle et al., Nucl. Phys. B, Proc. Suppl. 106, 177 (2002).

[9] P. A. Boyle, C. Jung, and T. Wettig (QCDOC), arXiv:heplat/0306023.

[10] S. Aoki, Phys. Rev. D 33, 2399 (1986).

[11] S. Aoki, Phys. Rev. Lett. 57, 3136 (1986).

[12] S. Aoki and Y. Taniguchi, Phys. Rev. D 65, 074502 (2002).

[13] While this paper addresses "nonquenched" physics with three dynamical flavors, the dynamical quark masses applied to surface states in our simulations are not related to the value of $M_{5}$ used in the domain wall construction, so it is the properties of the quenched Aoki phase which are most relevant for our calculations.

[14] S. R. Sharpe and R. L. Singleton, Jr., Phys. Rev. D 58, 074501 (1998).

[15] S. Aoki et al. (CP-PACS), Nucl. Phys. B, Proc. Suppl. 106, 718 (2002).

[16] M. Golterman and Y. Shamir, Phys. Rev. D 68, 074501 (2003).

[17] M. Golterman, Y. Shamir, and B. Svetitsky, Phys. Rev. D 71, 071502 (2005).

[18] M. Golterman, Y. Shamir, and B. Svetitsky, Phys. Rev. D 72, 034501 (2005).

[19] B. Svetitsky, Y. Shamir, and M. Golterman, Proc. Sci., LAT2005 (2006) 129.

[20] H. Neuberger, Phys. Rev. D 57, 5417 (1998).

[21] Y. Kikukawa and T. Noguchi, arXiv:hep-lat/9902022.

[22] P. Hernandez, K. Jansen, and M. Lüscher, Nucl. Phys. B552, 363 (1999).

[23] A. Borici, Nucl. Phys. B, Proc. Suppl. 83, 771 (2000).

[24] R. G. Edwards and U. M. Heller, Phys. Rev. D 63, 094505 (2001).

[25] A. Borici, arXiv:hep-lat/9912040.

[26] D. J. Antonio et al. (RBC and UKQCD), Proc. Sci., LAT2005 (2006) 141.

[27] N. Yamada et al. (JLQCD), Proc. Sci. LAT2006 (2006) 060.

[28] N. Christ (RBC and UKQCD), Proc. Sci., LAT2005 (2006) 345.

[29] Y. Shamir, Nucl. Phys. B406, 90 (1993).

[30] R. Narayanan and H. Neuberger, Nucl. Phys. B443, 305
(1995).

[31] For example, in the left-hand factor, the wrong-chirality operators, $P_{R} \hat{a}_{x}$ and $\hat{a}^{\dagger} P_{R}$, do not appear in the operator expression $\mathcal{O}_{L}\left[P_{L} q, \bar{q} P_{R}\right]$ and annihilate the state $\left\langle 0_{S}\right|$, and hence can be dropped for the present $m_{f}=0$ case.

[32] T. Blum et al., Phys. Rev. D 69, 074502 (2004).

[33] Y. Aoki et al., Phys. Rev. D 69, 074504 (2004).

[34] Y. Aoki et al., Phys. Rev. D 72, 114505 (2005).

[35] Y. Iwasaki, Nucl. Phys. B258, 141 (1985).

[36] Y. Iwasaki and T. Yoshie, Phys. Lett. 143B, 449 (1984).

[37] T. Takaishi, Phys. Rev. D 54, 1050 (1996).

[38] P. de Forcrand et al. (QCD-TARO), Nucl. Phys. B577, 263 (2000).

[39] S. A. Gottlieb, W. Liu, D. Toussaint, R. L. Renken, and R. L. Sugar, Phys. Rev. D 35, 2531 (1987).

[40] M. A. Clark and A. D. Kennedy, Phys. Rev. Lett. 98, 051601 (2007).

[41] M. A. Clark and A. D. Kennedy, Phys. Rev. D 75, 011502 (2007).

[42] D. J. Antonio et al. (UKQCD), Proc. Sci., LAT2005 (2006) 080.

[43] D. J. Antonio et al. (RBC), Proc. Sci., LAT2005 (2006) 098.

[44] D. J. Antonio et al., Phys. Rev. D 75, 114501 (2007).

[45] R. G. Edwards and B. Joo (SciDAC), Nucl. Phys. B, Proc. Suppl. 140, 832 (2005).

[46] P. Boyle, http://www.ph.ed.ac.uk/ paboyle/bagel/Bagel. html.

[47] D. J. Antonio et al. (RBC and UKQCD), Proc. Sci., LAT2005 (2006) 135.

[48] http://www.ph.ed.ac.uk/ paboyle/QCD/5dmode.mpg.

[49] K. Hashimoto, T. Izubuchi, and J. Noaki (RBC-UKQCD), Proc. Sci., LAT2005 (2006) 093.

[50] Y. Aoki et al., Phys. Rev. D 73, 094507 (2006).

[51] F. Berruto, R. Narayanan, and H. Neuberger, Phys. Lett. B 489, 243 (2000).

[52] R. G. Edwards, U.M. Heller, and R. Narayanan, Nucl. Phys. B535, 403 (1998).

[53] G. I. Egri, Z. Fodor, S. D. Katz, and K. K. Szabo, J. High Energy Phys. 01 (2006) 049.

[54] Z. Fodor, S. D. Katz, and K. K. Szabo, J. High Energy Phys. 08 (2004) 003.

[55] P. M. Vranas, arXiv:hep-lat/0001006.

[56] T. Izubuchi and C. Dawson (RBC), Nucl. Phys. B, Proc. Suppl. 106, 748 (2002).

[57] H. Fukaya et al. (JLQCD), Phys. Rev. D 74, 094505 (2006).

[58] C. Allton et al., Phys. Rev. D 76, 014504 (2007).

[59] R. Narayanan and H. Neuberger, Nucl. Phys. B412, 574 (1994).

[60] M. Lüscher, Commun. Math. Phys. 54, 283 (1977). 